\title{
Shedding Light on the Basis Set Dependence of the Minnesota Functionals: Differences Between Plane Waves, Slater Functions, and Gaussians
}

\author{
Martin P. Bircher, ${ }^{\circledR}$ Pablo López-Tarifa, and Ursula Rothlisberger*(i) \\ Laboratoire de Chimie et Biochimie Computationnelles, Ecole Polytechnique Fédérale de Lausanne, 1015 Lausanne, Switzerland
}

Supporting Information

ABSTRACT: The Minnesota family of exchange-correlation (xc) functionals are among the most popular, accurate, and abundantly used functionals available to date. However, their use in plane-wave based first-principles MD has been limited by their sparse availability. Here, we present an implementation of the M05, M06, and M11 families of xc functionals within a plane wave/pseudopotential framework allowing for a comprehensive analysis of their basis set dependence. While it has been reported that in Gaussian bases some members of the Minnesota family only converge slowly to the basis set limit, ${ }^{1}$ we show that converged energies can be conveniently obtained from plane waves if sufficiently dense integration meshes are

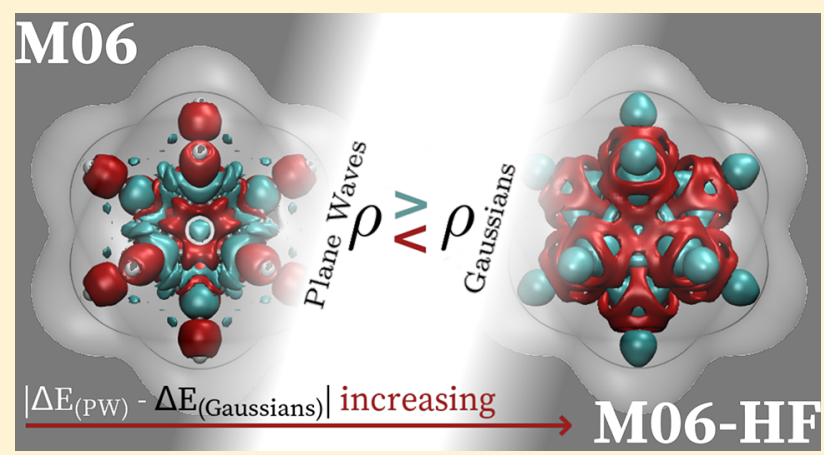
used. Based on the $\mathrm{HC} 7 / 11$ database, we assess the influence of basis set type on the calculation of reaction enthalpies and show that complete basis set values obtained in plane waves may occasionally differ notably from their atom-centered counterparts. We provide an analysis of the origin of these differences and discuss implications on practical usage.

\section{INTRODUCTION}

Density functional theory $(\mathrm{DFT})^{2}$ in its Kohn-Sham (KS) formulation $^{3}$ is one of the fundamental pillars of modern-day computational chemistry. Large systems of several hundreds of atoms can presently be treated. This is vital for the description of condensed matter systems with first-principles molecular dynamics (MD) simulations, in which thermodynamic properties can be obtained as time-averages at finite temperature. Even larger systems can be routinely treated using mixed quantum mechanics/molecular mechanics (QM/MM) ${ }^{4}$ approaches and with computational power ever increasing, the time scales that can be sampled are growing continuously, thus decreasing the statistical error. Powerful enhanced sampling methods have helped to reduce the time scales that have to be simulated, and at present, the error due to the sampling can become smaller than the error of the underlying potential energy surface (PES). The accuracy of the underlying exchange-correlation (xc) functional therefore becomes an increasingly dominant factor. The reliability of the underlying PES will not only be influenced by the choice of functional but also by the basis set used to expand the density. Some functionals may be particularly sensitive to the choice and size of basis. ${ }^{1,5,6}$ A plane wave expansion offers the intrinsic advantage of convergence control via a single parameter, the cutoff energy $E_{\text {cut }}$. A plane wave description is therefore ideally suited whenever a highly flexible, delocalized basis set is needed in order to obtain converged PES.
If KS-DFT has become abundantly used, then this is not at least due to the vast effort, persistence, and creativity that have been invested in the development of approximate forms for the unknown xc functional. This fundamental problem can be approached in many different ways. ${ }^{7,8}$ In some philosophies, functionals that contain as few free parameters as possible are generally preferred; the free parameters of such functionals are obtained from physical constraints and limits. 9 In another school of thought, a design that is directly fit to thermochemical data is considered nothing but an extension of basic physical constraints to the chemical world. ${ }^{10,11}$ Such an approach may be taken even further by obtaining free parameters not from atomic data but by resorting to larger databases of thermochemical and structural data. ${ }^{12,13}$ Even if the foundations of the different approaches to the problem may not be the same, they have ultimately been guided by the very same goal of improved accuracy and reliability.

A very prominent example of the latter philosophy is given by the widely used family of the Minnesota functionals, ${ }^{12-19}$ which have been developed by the Truhlar group. Many prominent functionals have been derived to offer one single functional that is as versatile as possible. Instead, the Minnesota philosophy aims to find the best possible performance for a given functional model and for a certain range of systems. Based on a given functional form, the free parameters

Received: September 2, 2018

Published: December 6, 2018 
are optimized according to different constraints: Certain parts of a test set may or may not be included in a given fit, and the functional form may or may not include an exact exchange contribution. Therefore, for every new generation of the Minnesota functionals, different variants have been developed, each with specific advantages in a certain regime but sharing a basic functional form. This approach has proven to be highly successful for many chemical problems. ${ }^{13,20}$ Even though the early members of the Minnesota family, the $M 05^{14,15}$ and M06 ${ }^{12,16,17,21}$ group of functionals, have since been superseded by more accurate models, the M06 family is still vastly popular in computational chemistry, as shown in Figure 1 by the rate of citations per year for the seminal M06 paper.

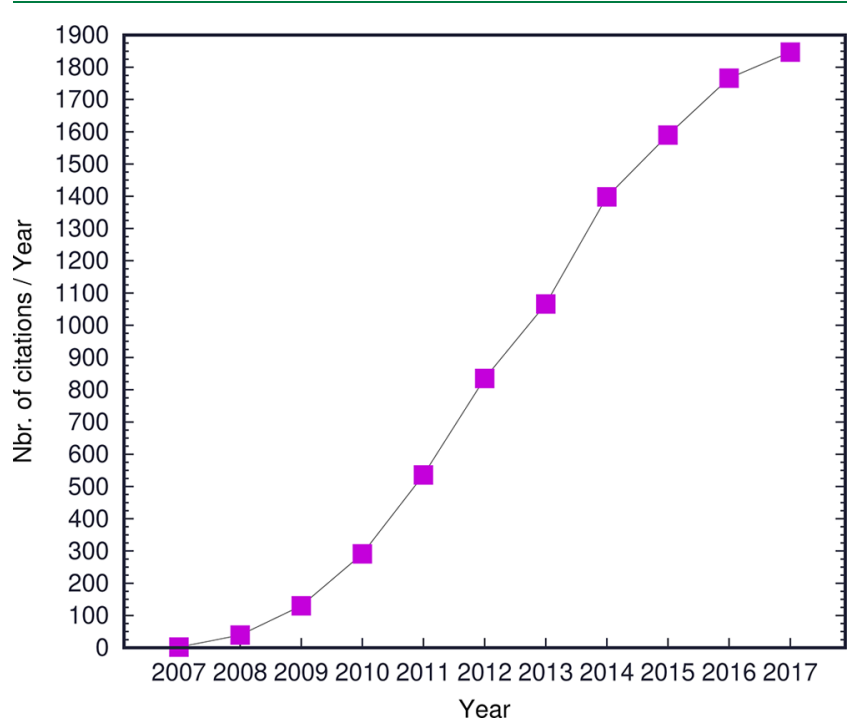

Figure 1. Citations per year for the seminal M06 paper $^{12}$ (source: webofknowledge). As of July 2018, the paper has accumulated a total of 10545 citations, making it the most cited of the Minnesota papers.

Along with the advent of empirically optimized functionals, several databases have been created which can be used both for the fitting of free parameters in functional development as well as for performance assessment. ${ }^{7,13,22,23}$ The HC7/11 database (Figure 2), for instance, gathers difficult hydrocarbon reaction enthalpies, ${ }^{12,22-24}$ including a set of particularly challenging isodesmic reactions. Truhlar and co-workers have shown that all functionals of the M06 family (i.e., M06-2X, M06-HF, M06-
L, and the parent M06 itself) outperform the other contemporary $\mathrm{xc}$ functionals, including the still very popular B3LYP; ${ }^{25,26}$ and similar trends were found for other data sets. $^{13,20}$

In the case of the CT7/04 set of charge-transfer dimers, ${ }^{22}$ the performance of the Minnesota functionals was either superior (M06-2X, M06-HF, M05) or at least on par (M06) with the best non-Minnesota xc functionals considered in the study $^{12,23}(\mathrm{~B} 97-3)$. $^{27}$

Despite such an encouraging performance, the underlying approach has also been met with scepticism. ${ }^{1,8}$ It has been argued that a fit that is not based on physical limits may deteriorate the formal qualities of a functional (however, all the Minnesota functionals are constrained to fulfill the UEG limit). Mardirossian et al. ${ }^{1}$ have shown that in an atom-centered basis of Gaussian functions, the energetics of some members of the Minnesota functional family converge remarkably slowly and may at first appear not to converge at all. By analyzing the inhomogeneity correction factors of the slowly converging functionals, they have shown that slow convergence correlates with either large correction factors for certain limits of the working function or with the occurrence of positive energy densities. The slow convergence of certain Minnesota functionals is also reflected in a high sensitivity toward basisset superposition errors (BSSE). ${ }^{1}$ Since the Minnesota functionals have been fit employing a specific basis set, the reference results can often only be obtained for the same (or a very similar) basis; while changing the "balance" of the basis set, or even approaching the basis set limit, may also change the energetics by up to a few $\mathrm{kcal} \mathrm{mol}^{-1}$. However, in many practical applications, functional/basis set combinations are benchmarked against accurate reference data ${ }^{13}$ and then used in that specific configuration. In these cases, a given functional/ basis set combination may be found to be accurate, even though the basis set limit values might show larger deviations with respect to the reference data than the combination adopted. From a theoretical point of view, however, the possibility of reaching convergence systematically and assessing the performance of a functional with a fully converged basis set is certainly desirable, in particular concerning the transferability of the results.

The study by Mardirossian et al. was conducted by expanding the density in atom-centered Gaussian functions. Inspired by the concept of overlapping atomic orbitals, atomcentered functions, and in particular Gaussians, are probably

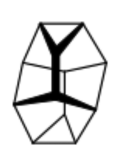

E1

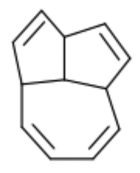

E22

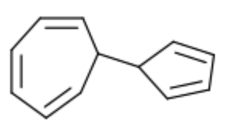

E31

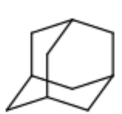

Adamantane

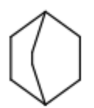

Bicyclo[2.2.2]octane

1

2

$\mathrm{E} 1 \rightarrow \mathrm{E} 22$

$\mathrm{E} 1 \rightarrow \mathrm{E} 31$

$3 \quad\left(\mathrm{CH}_{4}\right)_{4} \mathrm{CC}\left(\mathrm{CH}_{3}\right)_{3} \rightarrow n-\mathrm{C}_{8} \mathrm{H}_{18}$

$4 n-\mathrm{C}_{6} \mathrm{H}_{14}+4 \mathrm{CH}_{4} \rightarrow 5 \mathrm{C}_{2} \mathrm{H}_{6}$

$5 n-\mathrm{C}_{8} \mathrm{H}_{18}+6 \mathrm{CH}_{4} \rightarrow 7 \mathrm{C}_{2} \mathrm{H}_{6}$

6 adamantane $\rightarrow 3 \mathrm{C}_{2} \mathrm{H}_{4}+2 \mathrm{C}_{2} \mathrm{H}_{2}$

7 bicyclo[2.2.2] octane $\rightarrow \quad 3 \mathrm{C}_{2} \mathrm{H}_{4}+\mathrm{C}_{2} \mathrm{H}_{2}$

Figure 2. HC7/11 set of difficult hydrocarbon reactions. ${ }^{12}$ 
the most prominent choice of a basis set. However, the electron density may as well be expanded in other functional forms that need not necessarily be localized in space. Delocalized bases such as plane waves offer particular advantages beyond a simple control of convergence: Neither do Pulay forces occur, ${ }^{28}$ nor are basis-set superposition effects of any concern. This comes at the price of having to pseudise ${ }^{29}$ the effect of the core electrons; but the error due to this procedure has been shown to be negligible for the vast majority of chemically relevant properties of main group elements. ${ }^{6,29}$ These features make plane waves not only intrinsically suitable for first-principles molecular dynamics but also for the calculation of quantities that are sensitive to basisset superposition or that require multiple long-range functions. In a plane wave basis, the convergence of some property is monitored by increasing the cutoff energy of the highestfrequency reciprocal space vector. This makes plane waves ideally suited for the assessment of xc functional convergence, since the basis can be systematically increased until the basis set limit is attained. These features enable systematic convergence studies of $\mathrm{xc}$ functionals that may be difficult to converge in atom-centered bases.

Despite their popularity, availability of the Minnesota functionals in plane wave codes is scarce, being mostly limited to the (semi)local declinations of the functionals. ${ }^{30,31}$ Here, we present a comprehensive numerical analysis of an implementation of the M05, M06, M08, and M11 members of the Minnesota family in a plane wave/pseudopotential framework. Our implementation in the CPMD code makes Minnesota functionals available for routine plane wave calculations without the computational overhead (up to 20\%) due to external libraries.

The text is organized as follows: First, we give a short overview of the functional forms of the M05, M06, M08, and M11 families, followed by a brief description of the plane-wave specific details of our implementation. We then provide a comprehensive assessment of the basis set convergence in plane waves, which will be discussed at the example of the HF dimer. This member of the CT7/04 database had also been used by Mardirossian et al. in their convergence analysis in Gaussian bases, ${ }^{7}$ making a direct comparison to their results possible. Particular emphasis will be put on the functionals that Mardirossian et al. have identified as slowly converging. We will then show that using standard ratios between density and orbital cutoff values, for a certain subset of the functionals considered here, no systematic convergence is reached in a plane wave basis and that it is possible to systematically resolve this convergence issue by increasing the ratio between density and wave function cutoff. By including this additional parameter in the convergence analysis, the energetics of all functionals can be analyzed straightforwardly in a plane wave setup. Based on the possibility of obtaining converged energetics, we will then compare the influence of basis set type on the reaction enthalpies of hydrocarbons at the example of the HC7/11 database. Values will be compared between our plane wave implementation, two Gaussian basis sets commonly employed with the Minnesota family (aug-cc-pVTZ ${ }^{32}$ and 6$311+\mathrm{G}(2 \mathrm{df}, 2 \mathrm{p}))^{33}$ as well as two polarized Slater bases (TZ2P and ATZ2P). ${ }^{34}$ Slater functions exhibit an exact $r^{-1}$ decay, which makes it possible to assess the effects of long-range decay and basis set delocalization separately. This analysis will reveal that the range-separated functionals M11 and M11-L are particularly sensitive to the choice of basis and that converged energy differences obtained from plane waves or Slater functions may differ from their Gaussian equivalents, stressing the importance of a fully flexible basis. We will then see that results for M06-2X and M06-HF can deviate significantly from the values obtained in both Gaussian and Slater bases. We will show that these deviations can be tracked down by analyzing the exchange energy density $\epsilon_{x}(\mathbf{r})$, illustrating that the analytical form of the M06 family can lead to particular electron density differences between plane waves and Gaussian functions. Finally, we will provide a short discussion of the implication of our findings on the run time of plane wave calculations using the Minnesota family and suggest approaches to reduce the computational overhead.

\section{THEORY}

2.1. The Minnesota Functionals in a Nutshell. The first generation of the Minnesota family comprises the meta-hybrid functionals $\mathrm{M}^{14}$ and $\mathrm{M} 05-2 \mathrm{X},{ }^{15}$ as well as their direct successors $\mathrm{M}^{12},{ }^{12} \mathrm{M} 06-2 \mathrm{X},{ }^{12} \mathrm{M} 06-\mathrm{HF},{ }^{17}$ and the fully (semi)local meta-GGA M06-L. ${ }^{16}$ The semilocal terms will therefore depend on the spin-density $\rho_{\sigma}$, its gradient $\left|\nabla \rho_{\sigma}\right|$, and the spin-kinetic energy density, $\tau_{\sigma}=\sum_{i}^{o c c}\left|\nabla \psi_{i}^{(\sigma)}\right|^{2}$, calculated over all occupied generalized Kohn-Sham spin-orbitals $\psi_{i}^{(\sigma)}$. For notational simplicity, the implicit dependence of these functions on the Cartesian coordinates $\mathbf{r} \in \mathbb{R}^{3}$ will be omitted. In their most general form, the expressions for exchange and correlation energy for the M05 and M06 family read

$$
\begin{aligned}
& E_{\mathrm{x}}^{\mathrm{M} 05 / 6}=\alpha E_{\mathrm{HFX}}+(1-\alpha) \sum_{\sigma} \int_{\mathbb{R}^{3}} \epsilon_{\mathrm{x}, \sigma}^{\mathrm{UEG}}\left[F_{\mathrm{x}, \sigma}^{\mathrm{PBE}}\left[\rho_{\sigma}, \nabla \rho_{\sigma}\right] f\left(\omega_{\sigma}\right)\right. \\
& \left.\quad+h_{x}\left(x_{\sigma}, z_{\sigma}\right)\right] \\
& E_{\mathrm{c}}^{\mathrm{M} 05 / 6}=\int_{\mathbb{R}^{3}} \epsilon_{\mathrm{c}, \alpha \beta}^{\mathrm{UEG}}\left[g_{\alpha \beta}\left(x_{\alpha \beta}, z_{\alpha \beta}\right)+h_{\alpha \beta}\left(x_{\alpha \beta}, z_{\alpha \beta}\right)\right] \\
& \quad+\sum_{\sigma} \int_{\mathbb{R}^{3}} \epsilon_{\mathrm{c}, \sigma \sigma}^{\mathrm{UEG}}\left[g_{\sigma \sigma}\left(x_{\sigma}, z_{\sigma}\right) D_{\sigma}\left(x_{\sigma}, z_{\sigma}\right)+h_{\sigma \sigma}\left(x_{\sigma}, z_{\sigma}\right)\right]
\end{aligned}
$$

where $\epsilon_{\mathrm{x}}$ and $\epsilon_{\mathrm{c}}$ denote the exchange and correlation energy density of the uniform electron gas (UEG), respectively. The exchange enhancement factor is a linear combination of the gradient-dependent $\mathrm{PBE}$ exchange ${ }^{9}$ term $F_{\mathrm{x}, \sigma}^{\mathrm{PBE}}\left[\rho_{\sigma}, \nabla \rho_{\sigma}\right]$, weighted by a parametrized function $f\left(\omega_{\sigma}\right)$ (which, through $\omega_{\sigma}$, depends on $\rho_{\sigma}$ and $\tau_{\sigma}$ ) and a reparametrization of the VS98 ${ }^{35}$ correction term, $h_{\mathrm{x}}\left(x_{\sigma}, z_{\sigma}\right)$ (which, through $x_{\sigma}$ and $z_{\sigma}$, depends in itself on $\rho,|\nabla \rho|$, and $\tau)$. The form of $f\left(\omega_{\sigma}\right)$ is common to all Minnesota functionals and reads

$$
f\left(\omega_{\sigma}\right)=\sum_{i=0}^{m} a_{i} \omega_{\sigma}^{i}
$$

where the $\left\{a_{i}\right\}$ are empirically determined weights and

$$
\begin{aligned}
& \omega_{\sigma}\left(t_{\sigma}\right)=\frac{\left(t_{\sigma}-1\right)}{\left(t_{\sigma}+1\right)} \\
& t_{\sigma}\left(\rho_{\sigma}, \tau_{\sigma}\right)=\frac{3}{10}\left(6 \pi^{2}\right)^{2 / 3} \frac{\rho_{\sigma}^{5 / 3}}{\tau_{\sigma}}
\end{aligned}
$$

The correlation functional is based on the Stoll ansatz; ${ }^{36}$ the correlation energy of the uniform electron gas is corrected by a linear combination of the kinetic-energy density dependent self-interaction correction factor $D_{\sigma}\left(x_{\sigma}, z_{\sigma}\right)$ on one hand, and the VS98 correlation integrand $h_{\sigma \sigma}\left(x_{\sigma}, z_{\sigma}\right)$ on the other hand. Whereas the free parameters in the latter are reoptimized, 
$D_{\sigma}\left(x_{\sigma}, z_{\sigma}\right)$ is weighted by a parametrized expression of the form:

$$
g_{\sigma \sigma}\left(x_{\sigma}, z_{\sigma}\right)=\sum_{i=0}^{n} c_{\sigma \sigma}^{(i)}\left(\frac{\gamma_{\sigma \sigma} x_{\sigma}{ }^{2}}{1+\gamma_{\sigma \sigma} x_{\sigma}{ }^{2}}\right)^{i}
$$

In all functionals of the M05 family, the terms due to VS98 are naught; the constants in the parametrized functions differ for all functionals. A detailed description of the assembly of the functionals and the parametrized functions $f\left(\omega_{\sigma}\right), h_{\mathrm{x}}\left(x_{\sigma}, z_{\sigma}\right)$, $g_{\sigma \sigma}\left(x_{\sigma}, z_{\sigma}\right)$, as well as the terms in $D_{\sigma}\left(x_{\sigma}, z_{\sigma}\right)$ and $h_{\sigma \sigma}\left(x_{\sigma}, z_{\sigma}\right)$ are given in the Supporting Information, along with a description of some notational intricacies.

The subsequent generation of functionals, $\mathrm{M}^{21}$ and $\mathrm{M} 11,{ }^{18,19}$ are again based on a linear combination of existing GGA enhancement factors. Contrary to their predecessors, definitions based on the spin-unpolarized density $\rho=\rho_{\alpha}+\rho_{\beta}$ are used throughout. The meta-hybrids of the M08 family, M08-SO and M08-HX, are constructed according to $E_{\mathrm{x}}^{\mathrm{M} 08}=$ $\alpha E_{\mathrm{HFX}}+(1-\alpha) E_{\mathrm{x}, \mathrm{loc}}^{\mathrm{M} 08 / 11}$, and the semilocal term alone reads

$$
E_{\mathrm{x}, \mathrm{loc}}^{\mathrm{M} 08 / 11}=\int_{\mathbb{R}^{3}} \epsilon_{\mathrm{x}}^{\mathrm{LDA}}\left[f_{1}(\omega) F_{\mathrm{x}}^{\mathrm{PBE}}[\rho, \nabla \rho]+f_{2}(\omega) F_{\mathrm{x}}^{\mathrm{RPBE}}[\rho, \nabla \rho]\right]
$$

where $F_{\mathrm{x}}^{\mathrm{PBE}}$ and $F_{\mathrm{x}}^{\mathrm{RPBE}}$ are the $\mathrm{PBE}$ and $\mathrm{RPBE}^{37}$ exchange enhancement factors, respectively. For the M08 functionals, the $\tau$-dependent weights $f_{i}(\omega)$ are defined analogously to the M05 and M06 family. A generalization to spin is trivial using the spin-scaling relations. The form of the $f_{i}(\omega)$ is also used for the correlation functional, where the Stoll ansatz is abandoned in favor of the expression:

$$
E_{\mathrm{c}}^{\mathrm{M} 08}=\int_{\mathbb{R}^{3}} \rho\left[e_{\mathrm{C}}^{\mathrm{LSDA}}(\rho, \zeta) f_{3}(\omega)+H^{\mathrm{PBE}}(\rho, \nabla \rho, \zeta, t) f_{4}(\omega)\right]
$$

We have introduced the function $\zeta=\left(\rho_{\alpha}-\rho_{\beta}\right) / \rho$ for the spin polarization, $e_{\mathrm{C}}^{\mathrm{LSDA}}$ is the (spin-dependent) correlation energy per unit density in the Perdew-Wang form, ${ }^{38}$ and $H^{\mathrm{PBE}}$ is the $\mathrm{PBE}$ correction to the correlation energy.

The M11 generation introduces range-separated exchange $e^{39-41}$ by suitably modifying the M08 functional form: $E_{\mathrm{x}}^{\mathrm{M} 11}=E_{\mathrm{x}, \mathrm{SR}}^{\mathrm{M} 11}+E_{\mathrm{x}, \mathrm{LR}}^{\mathrm{M} 11}$. In the range-separated meta-hybrid M11, $E_{\mathrm{x}, \mathrm{LR}}^{\mathrm{M} 11}$ is obtained from an exact exchange integral employing a long-range Coulomb operator $\hat{W}$ of the form $\hat{W}_{\mathrm{LR}}=\sum_{i} \sum_{i \neq j}[\alpha$ $\left.+\beta \operatorname{erf}\left(\mu r_{i j}\right)\right] / r_{i j}$ with three range-separation parameters $\alpha, \beta$, and $\mu$. In the case of the fully (semi)local dual-range functional $\mathrm{M} 11, E_{\mathrm{x}, \mathrm{LR}}^{\mathrm{M} 11}$ is treated with the functional form of $E_{\mathrm{x}, \mathrm{loc}}^{\mathrm{M} 08 / 11}$ by using a suitable generalization of the form $\epsilon_{\mathrm{x}}^{\mathrm{LR}}=\epsilon_{\mathrm{x}}^{\mathrm{LDA}}(1-G(\rho$, $\alpha, \beta, \mu))$, with the local short-range attenuation function $G(\rho$, $\alpha, \beta, \mu) .{ }^{41}$ In both M11 and M11-L, the short-range part itself is treated analogously to $E_{\mathrm{x}, \mathrm{LR}}^{\mathrm{M} 11}[\rho]$, with $e_{\mathrm{x}}^{\mathrm{SR}}$ replaced by the short-range form $\epsilon_{\mathrm{x}}^{\mathrm{SR}}=\epsilon_{\mathrm{x}}^{\mathrm{LDA}} G(\rho, \alpha, \beta, \mu) .^{41}$

2.2. Expanding the Electron Density in Plane Waves. At the $\Gamma$-point, the (real) Kohn-Sham orbitals can be expanded in a plane wave basis of the form: ${ }^{29}$

$$
\psi_{i}(\mathbf{r})=\sum_{\mathbf{G}=0}^{\mathbf{G}_{\max }} \psi_{i}(\mathbf{G}) e^{i \mathbf{G} \cdot \mathbf{r}}
$$

where $\mathbf{r}, \mathbf{G}$ are real and reciprocal space vectors, respectively, and the $\left\{\psi_{i}(\mathbf{G})\right\}$ are the (Fourier) expansion coefficients of an orbital indexed by $i$. The length of the expansion is given by the highest-frequency $\mathbf{G}$-vector $\mathbf{G}_{\max }$ and is usually indicated with respect to its energy, the cutoff energy $E_{\text {cut }}$. In a discretized Cartesian basis, the $\psi_{i}(\mathbf{r})$ and $\psi_{i}(\mathbf{G})$ are mutual Fourier transforms. It is therefore straightforward to switch between representations at an $O(N(\log N))$ scaling using fast Fourier transforms (FFT). This is of particular advantage for the computation of the Coulomb potential: Since the Coulomb operator is diagonal in reciprocal space, $\Phi(\mathbf{G})=\frac{4 \pi}{\mathbf{G}^{2}}$, the corresponding potential is easily obtained from the reciprocal space density $\rho(\mathbf{G})$. The density itself is most conveniently constructed in real space:

$$
\rho(\mathbf{r})=\sum_{i} f_{i}\left|\psi_{i}(\mathbf{r})\right|^{2}
$$

where $f_{i}$ are occupation numbers and $\rho(\mathbf{G})$ is obtained via a FFT. However, due to the square in eq 10, the spectrum of the function $\rho$ will extend to higher-frequency $\mathbf{G}$-vectors than the initial $\psi$. This requires for a second cutoff energy to be introduced, $E_{\text {cut }}^{\rho}$, with a value of 4 being analytically sufficient to guarantee correspondence between $\psi$ and $\rho$. The ratio between $E_{\text {cut }}^{\rho}$ and $E_{\text {cut }}$ is commonly referred to as the dual:

$$
\xi=\frac{E_{\text {cut }}^{\rho}}{E_{\text {cut }}}
$$

Choosing a value of $\xi$ larger than the default value of $r$ results in real-space representations of $\rho$ which is Fourier-interpolated with respect to the default grid. This makes it possible to arbitrarily increase the resolution of the mesh without introducing higher-frequency components in the description of $\psi$, therefore minimizing numerical noise.

2.3. Range-Separated and Screened Exchange in Plane Waves. In plane waves, the exact exchange of the Kohn-Sham determinant is usually calculated in reciprocal space from $^{42}$

$$
E_{\mathrm{x}}^{0}[\rho]=-\frac{1}{2} \sum_{i}^{N_{\mathrm{b}}} \sum_{j}^{N_{\mathrm{b}}} \sum_{\mathbf{G}}^{\mathbf{G}_{\max }} \Phi(\mathbf{G})\left|\rho_{i j}(\mathbf{G})\right|^{2}
$$

where $\Phi(\mathbf{G})$ denotes the reciprocal space (i.e., Fourier series) representation of a (generic) Coulomb operator $\hat{W}$ and $\left.\rho_{i j}(\mathbf{G})=\mathcal{F} \psi_{i}^{*}(\mathbf{r}) \psi_{j}(\mathbf{r})\right]$ are the Fourier transforms of pair densities constructed from $N_{\mathrm{b}}$ occupied Kohn-Sham orbitals. In a fully periodic setup and within a discrete representation of $\mathbf{G}, E_{\mathrm{x}}^{0}[\rho]$ exhibits an (integrable) divergence at $\mathbf{G}=0 . \Phi(\mathbf{G})$ therefore needs to be appropriately modified. ${ }^{43,44}$ For the conventional Coulomb operator $\hat{W}=\sum_{i j} \frac{1}{r_{i j}}$, based on an initial procedure by Gygi and Baldereschi, ${ }^{43}$ Broqvist et al. ${ }^{44}$ have suggested to write

$$
\Phi(\mathbf{G})= \begin{cases}\frac{1}{\Omega} \frac{4 \pi}{\mathbf{G}^{2}} & \text { for } \mathbf{G} \neq 0 \\ \chi(0) & \text { for } \mathbf{G}=0\end{cases}
$$

where $\Omega$ denotes the supercell volume and the screening function $\chi(0)$ is obtained as the $\lim _{\gamma \rightarrow 0}$ from

$$
\chi(\gamma)=\left[\frac{1}{\sqrt{\pi \gamma}}-\frac{4 \pi}{\Omega} \sum_{\mathbf{G}} \frac{\mathrm{e}^{-\gamma \mathbf{G}^{2}}}{\mathbf{G}^{2}}\right]
$$

It is straightforward to show that for a Coulomb attenuated operator $\hat{W}_{\mathrm{lr}}=\frac{\alpha+\beta \operatorname{erf}\left(\mu r_{i j}\right)}{r_{i j}}$, we have that ${ }^{6}$ 


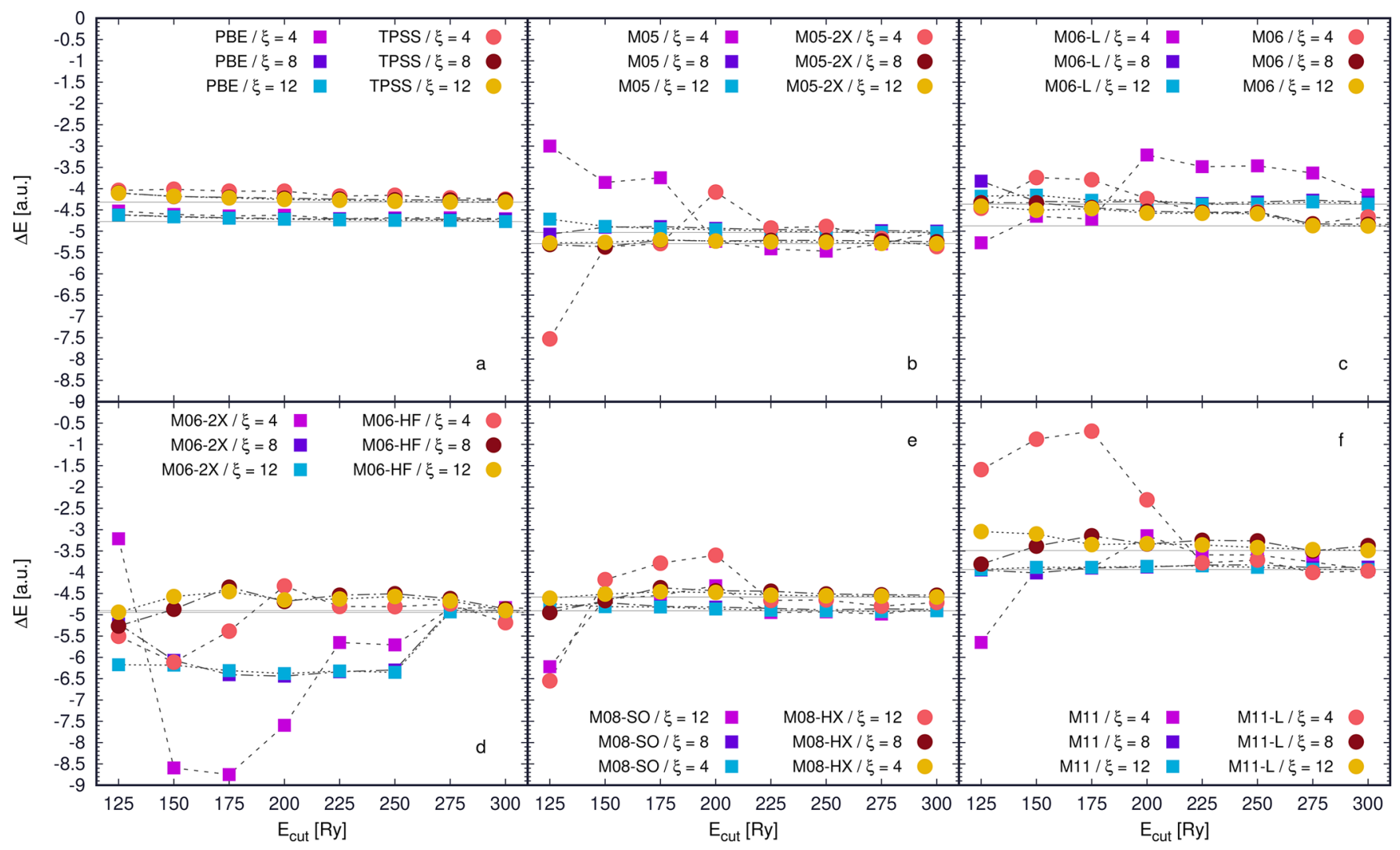

Figure 3. Convergence of the binding energy $\Delta E$ of a HF dimer, grouped according to functionals, with respect to cutoff energy $E_{\text {cut }}$ and $\xi$. Converged binding energy values are illustrated with a line.

$$
\Phi_{\mathrm{lr}}(\mathbf{G})=\left\{\begin{array}{cc}
\frac{1}{\Omega} \frac{4 \pi}{\mathbf{G}^{2}}\left[\alpha+\beta \mathrm{e}^{-G^{2} / 4 \mu^{2}}\right] & \text { for } \mathbf{G} \neq 0 \\
\alpha \chi(0)+\beta \chi\left(\frac{1}{4 \mu^{2}}\right) & \text { for } \mathbf{G}=0
\end{array}\right.
$$

Our implementation of the range-separated Minnesota family members make use of eq 15 , a derivation of which is available in the literature. ${ }^{6,44}$ An in-depth discussion of the effect of a screened term at $\mathbf{G}=0$ has been provided in refs 43,44 , and 6 , with results of plane wave/pseudopotential calculations providing good agreement with atom-centered, nonperiodic basis sets.

\section{COMPUTATIONAL DETAILS}

Plane wave calculations were carried out using the CPMD code. ${ }^{45}$ Hard Goedecker-Teter-Hutter $(\mathrm{GTH})^{46}$ pseudopotentials have been used in order to ensure maximum transferability. Hartree-Fock exchange energies, ${ }^{47}$ where not otherwise stated, were obtained using a constant, default $\xi=4$, whereas $\xi$ used in the calculation of $\tau, \nabla \rho, \rho$, and the xc energy were set to different values. These are reported in the Results and Discussion, along with orbital cutoff energies $E_{\text {cut }}$. The Poisson equations of the periodic images were decoupled using the algorithm by Martyna and Tuckerman. ${ }^{48}$ The orthorhombic supercell for the HF dimer spanned $20 \times 15 \times 10 \AA^{3}$, whereas the supercells for the reactions of the $\mathrm{HC} / 11$ database were cubic of dimension $30^{3} \AA^{3}$. In order to rule out basis set convergence issues for the M06-2X functional for reactions 1 and 2, reaction energies were also calculated in a
$20^{3} \AA^{3}$ supercell, using a cutoff energy of $275 \mathrm{Ry}$ and $\xi=12$ for both local and Hartree-Fock contributions.

Calculations employing a Slater basis were performed using the $\mathrm{ADF}^{49-51}$ program package and the TZ2P and ATZ2P basis sets, ${ }^{34}$ adding diffuse fitting functions for the RI calculations and eliminating linearly dependent basis functions using a threshold of $10^{-4}$. The xc energy and potentials were calculated using libxc" ${ }^{52}$ on a very fine Becke grid" ("excellent quality" in ADF jargon). Calculations using Gaussian basis sets were carried out using the Gaussian $16^{54}$ suite of programs, a tight convergence criterion on the Kohn-Sham orbitals and a superfine integration grid.

\section{RESULTS AND DISCUSSION}

4.1. Total Energy of a HF Dimer. In the following, we will provide an analysis of the convergence of the M05 to M11 families of functionals in a plane wave basis. First, we will consider the conventional case at fixed $\xi=4$ and increasing $E_{\text {cut. }}$ We will then perform the same analysis at increasing values of $\xi$ in order to assess the effect of a denser mesh.

4.1.1. Convergence in Plane Waves. The convergence behavior of the functionals considered here with respect to the energy cutoff $E_{\text {cut }}$ and using a standard $\xi=4$ is illustrated in Figure 3 with an example of the binding energy of an HF dimer. A reference value for every functional, obtained at a cutoff of $300 \mathrm{Ry}$ and $\xi=12$, is illustrated as a straight line.

Figure $3 \mathrm{a}$ shows the convergence of the PBE and TPSS ${ }^{55} \mathrm{xc}$ functionals, which will serve as an example for standard generalized gradient approximation (GGA) and meta-generalized gradient approximation (MGGA) functionals, respectively. Even at the lowest cutoff energy considered here, 125 
Ry, the energy is converged to less than $0.3 \mathrm{kcal} \mathrm{mol}^{-1}$, which is already within chemical accuracy. Values at $225 \mathrm{Ry}$ are essentially converged in a sub $0.1 \mathrm{kcal} \mathrm{mol}^{-1}$ regime. In both cases, the energies at lower cutoff values are an upper bound to the reference value. The situation is fundamentally different for the Minnesota functionals studied here. In Figure $3 b$, the oldest members of the group, M05 and M05-2X, are shown. In both cases, the reference energy can only be reached at a cutoff value of $300 \mathrm{Ry}$, with deviations at $125 \mathrm{Ry}$ reaching the $3 \mathrm{kcal}$ $\mathrm{mol}^{-1}$ range. In both cases, the unconverged energies do not serve as an upper bound for the converged values. A similar behavior is observed in Figure $3 c$ for the M06-L functional, although the maximum deviation is less than $1.5 \mathrm{kcal} \mathrm{mol}^{-1}$. The spread of the values is much lower for M06, where deviations $<0.3 \mathrm{kcal} \mathrm{mol}^{-1}$ are reached at $200 \mathrm{Ry}$.

The situation improves for M06-HF in panel 3d, where deviations only narrowly exceed $1 \mathrm{kcal} \mathrm{mol}^{-1}$ and lie within a $0.3 \mathrm{kcal} \mathrm{mol}^{-1}$ range from $225 \mathrm{Ry}$ onward. Values for M06-2X, on the other hand, are most erratic and vary by up to almost 3 $\mathrm{kcal} \mathrm{mol}^{-1}$ for certain cutoff values. For the two members of the M08 family shown in Figure 3e, both M08-SO and M08HX converge around $225 \mathrm{Ry}$, even though deviations at $125 \mathrm{Ry}$ are larger than for the M06 family. Deviations are again larger for M11 and M11-L shown in Figure 3f, with maximum deviations of $2 \mathrm{kcal} \mathrm{mol}^{-1}$, second only to the M05 family.

When discussing the basis set convergence in a Gaussian basis, several authors have stressed the importance of a fine integration $\operatorname{grid}^{1,56,57}$ in order to obtain accurate values for most Minnesota functionals (however, Mardirossian et al. have shown that the size of the integration grid may not necessarily constitute the principal cause of slow convergence with respect to the basis set $\left.\operatorname{size}^{1}\right)$. In plane waves, while an increase in cutoff energy $E_{\text {cut }}$ implies a finer mesh on which the xc energy is evaluated, the high frequency components in reciprocal space may also introduce further noise. A finer integration grid can be obtained by increasing the value of $\xi$. Performing the convergence analysis at higher values of the dual $\xi$ therefore allows one to assess the influence of grid granularity on the energy convergence.

Figure 3 shows a clear improvement of the convergence behavior of the Minnesota functionals for higher values of $\xi$. For the reference functionals in Figure 3a, the influence of an increased mesh is far below chemical accuracy. Even for the more sensitive TPSS functional, deviations between different values of $\xi$ do not exceed $0.2 \mathrm{kcal} \mathrm{mol}^{-1}$. For both PBE and TPSS, convergence within a sub $0.1 \mathrm{kcal} \mathrm{mol}^{-1}$ interval can be reached at $175 \mathrm{Ry}$ when using $\xi \geq 8$. Values obtained using $\xi$ of 8 and 12 are virtually indistinguishable for both functionals. The convergence behavior of the Minnesota functionals in Figure $3 \mathrm{~b}-\mathrm{f}$ substantially improves upon increasing $\zeta$. For both $\xi$ of 8 and 12, all energies except M06-HF, M06-2X, and M11$\mathrm{L}$ lie within the $a<0.3 \mathrm{kcal} \mathrm{mol}^{-1}$ interval starting from 150 to $175 \mathrm{Ry}$ and are close to within a sub $0.1 \mathrm{kcal} \mathrm{mol}^{-1}$ range from $200 \mathrm{Ry}$ on. The convergence behavior is therefore very similar to the PBE and TPSS functionals indicating that it is not the high-frequency components in the wave function expansion that are needed for accurate energetics but rather a very fine mesh for the density. These observations are in line with the common requirement imposed on the integration grid for Minnesota functionals in atom-centered bases. Increasing $\xi$ further from 8 to 12 results in changes of energetics that are negligible. The remaining outliers are M06-2X, M06-HF, and M06-L. For M06-HF, values for $\xi$ of 8 or 12 remain within a
$0.3 \mathrm{kcal} \mathrm{mol}^{-1}$ range. For cutoff energies lower than $200 \mathrm{Ry}$, results obtained from $\xi=8$ deviate visibly from those obtained at $\xi=12$. The only other example of this behavior is M11-L, which overall behaves very similarly to M06-HF for higher values of $\xi$ and is therefore slightly less well-behaved than many of the earlier-generation Minnesota functionals studied here. Still, due to the narrow spread of the energies once a higher $\xi$ is used, this is not expected to result in practical problems. The least well-behaved outlier is M06-2X, where binding energies abruptly change at 275 Ry. Only from this value onward do the energies remain within a very narrow range and appear to be properly converged. We have verified the stability of the M06-2X results by increasing the value of $\xi$ $=20$, which did not alter the convergence behavior, nor did it have any significant influence on the binding energies. It therefore appears that, in addition to a suitably fine integration grid, M06-2X needs an increased amount of high-frequency components in the wave function expansion in order to account for the binding of the HF dimer.

For practical applications, energy differences can therefore be converged straightforwardly, provided that the value of $\xi$ is increased above the standard of 4 . For chemical accuracy, a value of 8 has proven sufficient for the system considered here. This makes it possible to obtain reference values for the Minnesota functionals in a fully nonlocal basis, enabling studies free of basis-set superposition errors and independent of the balance of basis functions and integration grids employed. In the case of M06-2X, at least for the weakly bound HF dimer studied here, particular attention has to be paid to the choice of integration grid and cutoff.

The substantial improvement of convergence behavior when increasing $\xi$ from 4 to 8 illustrates yet once more the need for a dense enough mesh. Our observations suggest that the cutoff of the basis set is as straightforwardly controlled as it is the case of conventional functionals (with the exception of M06-2X, which requires particular attention). Instead, for a basis of a given size, it is the integration mesh of the xc term that is the most crucial ingredient. This is an unconventional observation in a plane wave basis, since commonly and as reported here for PBE and TPSS, convergence is reached straightforwardly by increasing the energy of the highest-frequency plane wave in the expansion at a default $\xi=4$. However, this exceptional behavior of the Minnesota functionals is in line with the requirements for very dense integration grids that are recommended in Gaussian bases. ${ }^{56}$ In contrast to commonly employed Gaussian bases and the observations by Mardirossian et al. we can rule out any influence of the balance of the exponents of the basis set on the final result, implying that for a given pseudopotential and given an appropriately dense mesh, the convergence behavior of the Minnesota functionals does not differ from that of other meta-GGA functionals. The flexibility of a plane-wave based description therefore makes it possible to obtain truly converged energetics, which can serve as reference values for calculations carried out in other (atomcentered) basis sets.

4.1.2. Comparison to Atom-Centered Bases. Among the functionals studied, M05, M06-2X, M06-HF and, to a lesser extent, the M08 and M11 families are the most sensitive to changes in cutoff energies. Both M06-HF and M11-L are functionals that Mardirossian et al. have identified as particularly slowly convergent with respect to the Gaussian basis set size, unlike the M05 family and M06-2X, which were found to be comparably well-behaved. However, the 
Table 1. HF Dimer Binding Energy Calculated for the M05, M06, M08, and M11 Families of Minnesota Functionals in Different Basis Sets ${ }^{a}$

\begin{tabular}{|c|c|c|c|c|c|c|c|c|}
\hline \multirow[b]{2}{*}{ functional } & \multicolumn{3}{|c|}{ plane waves } & \multicolumn{2}{|c|}{ Slater functions } & \multicolumn{3}{|c|}{ Gaussian functions } \\
\hline & $\xi=4$ & $\xi=8$ & $\xi=12$ & TZ2P & ATZ2P & aug-cc-pVTZ & $6-311++G(2 d f, 2 p)$ & limit \\
\hline M05 & -5.02 & -5.00 & -5.03 & -4.98 & -4.95 & -4.99 & -5.36 & -4.96 \\
\hline M05-2X & -5.36 & -5.25 & -5.30 & -5.12 & -5.08 & -5.06 & -5.18 & -5.04 \\
\hline M06 & -4.74 & -4.84 & -4.88 & -4.20 & -4.24 & -4.40 & -4.71 & -4.44 \\
\hline M06-2X & -4.87 & -4.90 & -4.94 & -4.90 & -4.88 & -4.95 & -5.13 & -4.87 \\
\hline M06-HF & -5.18 & -4.86 & -4.90 & -4.43 & -4.48 & -4.71 & -5.01 & -5.24 \\
\hline M06-L & -4.15 & -4.32 & -4.37 & -4.49 & -4.52 & -4.46 & -4.55 & -4.59 \\
\hline M08-HX & -4.71 & -4.54 & -4.58 & -4.69 & -4.70 & -4.94 & -5.14 & -4.74 \\
\hline M11 & -3.94 & -3.88 & -3.94 & -4.45 & -4.53 & -4.58 & -4.87 & -4.47 \\
\hline M11-L & -3.97 & -3.38 & -3.50 & -3.57 & -3.73 & -3.86 & -4.10 & -3.41 \\
\hline
\end{tabular}

${ }^{a}$ Results for aug-cc-pVTZ and a customized complete Gaussian basis (Limit) are reproduced from ref 1. Plane waves results were obtained at a wavefunction cutoff of $300 \mathrm{Ry}$. Atom-centered results are not Counterpoise corrected, in agreement with common procedures used in functional benchmarking and fitting.

convergence of M05 and M05-2X substantially improves once $\xi$ is increased resulting in a well-behaved approach to the converged limit (which has also been documented in an atomcentered basis of Gaussian functions at the example of a sufficiently fine integration grid). M06-L, however, appears to be much more well-behaved in plane waves than what was reported for a Gaussian basis.

Table 1 provides more insight into the basis-set sensitivity of the Minnesota family of functionals. Values of the HF dimer binding energy are reported for all three choices of dual considered here $(4,8,12)$. The converged values from plane waves at $\xi=12$ are compared to values obtained from the commonly used and popular aug-cc-pVTZ and 6-311+ $+\mathrm{G}(2 \mathrm{df}, 2 \mathrm{p})$ basis sets as well as the complete Gaussian basis set of ref 1 as a reference. In order to exclude possible effects due to the unphysical long-range decay and cusp behavior of Gaussian functions, results for a larger Slater basis set (TZ2P) and an augmented Slater basis (ATZ2P) are also given.

For both members of the M05 family, results across all bases agree well, with a maximum deviation of $0.26 \mathrm{kcal} \mathrm{mol}^{-1}$ between the converged plane wave result and the complete basis set limit obtained from Gaussian functions. Results obtained in a Slater basis do not deviate by more than $0.04 \mathrm{kcal}$ $\mathrm{mol}^{-1}$ from aug-cc-pVTZ and the limit value from ref 7; changes upon inclusion of an augmentation function are lower than $0.04 \mathrm{kcal} \mathrm{mol}^{-1}$. However, the binding energy obtained with $6-311++G(2 d f, 2 p)$, which is similar to the basis commonly used to benchmark the database that contains the $\mathrm{HF}$ dimer, deviates by about $0.4 \mathrm{kcal} \mathrm{mol}^{-1}$ from the reference value in the case of M05. As reported by Mardirossian et al., changes between the complete basis and aug-cc-pVTZ are negligible. For M05 and M05-2X, the overall agreement between the different bases is well within chemical accuracy.

The deviations are larger for some members of the M06 family. For the parent M06 itself, plane wave binding energies using $\xi=12$ exceed the reference value by $0.44 \mathrm{kcal} \mathrm{mol}^{-1}$. The values obtained in a Slater basis are by about $0.20 \mathrm{kcal}$ $\mathrm{mol}^{-1}$ lower than the reference value and are again insensitive to the addition of augmentation functions. Results from 6 to $311++\mathrm{G}(2 \mathrm{df}, 2 \mathrm{p})$ are closer to plane wave values than to the converged value in a Gaussian basis. For M06-2X, the agreement between plane waves, both Slater bases, aug-cc-
pVTZ, and the value from a complete Gaussian basis show excellent agreement, with a deviation of $\leq 0.10 \mathrm{kcal} \mathrm{mol}^{-1}$. The largest error with respect to the limit occurs again for 6-311+ $+\mathrm{G}(2 \mathrm{df}, 2 \mathrm{p})$, with a difference of $0.25 \mathrm{kcal} \mathrm{mol}^{-1}$. Given the rather erratic convergence behavior of this functional, these are encouraging results, indicating that once the requirements on grid and cutoff are met, the plane wave implementation accurately reproduces results from atom-centered bases. In the case of M06-HF, converged plane wave values differ by 0.34 $\mathrm{kcal} \mathrm{mol}^{-1}$ from the reference, which is smaller than the deviation that occurs for aug-cc-pVTZ $\left(0.53 \mathrm{kcal} \mathrm{mol}^{-1}\right)$. The value for $6-311++\mathrm{G}(2 \mathrm{df}, 2 \mathrm{p})$ is closer to the limit, while both augmented and nonaugmented Slater bases give deviations of up to $0.81 \mathrm{kcal} \mathrm{mol}^{-1}$. The situation is improved for the completely local M06-L, where the deviation between the converged plane wave value and the limit in a Gaussian basis is $0.22 \mathrm{kcal} \mathrm{mol}^{-1}$. The value for aug-cc-pVTZ is slightly closer (error of $0.13 \mathrm{kcal} \mathrm{mol}^{-1}$ ), whereas the two Slater bases and 6$311++\mathrm{G}(2 \mathrm{df}, 2 \mathrm{p})$ are closest to the value of the limit.

The situation is similar for the more recent M08 and M11 families. For M08-HX, the converged plane wave result differs by only $0.16 \mathrm{kcal} \mathrm{mol}^{-1}$, which is smaller than the deviations of aug-cc-pVTZ and $6-311++\mathrm{G}(2 \mathrm{df}, 2 \mathrm{p}) \quad(0.20$ and $0.40 \mathrm{kcal}$ $\mathrm{mol}^{-1}$, respectively). The binding energies obtained using TZ2P and ATZ2P show only a negligible deviation of $\leq 0.10$ $\mathrm{kcal} \mathrm{mol}^{-1}$. Plane wave deviations are largest for M11, where the converged plane wave binding energy differs by $0.51 \mathrm{kcal}$ $\mathrm{mol}^{-1}$ from the reference value. It should however be noted that this value is comparable to the deviations observed for Slater bases in the case of M06-HF and for 6-311++G(2df,2p) in the case of M06. Values obtained within a Slater basis and aug-cc-pVTZ are again closer to the reference (deviations of maximum $\left.0.11 \mathrm{kcal} \mathrm{mol}^{-1}\right)$, while $6-311++\mathrm{G}(2 \mathrm{df}, 2 \mathrm{p})$ is again the least accurate Gaussian basis, with an error of $0.40 \mathrm{kcal}$ $\mathrm{mol}^{-1}$. Errors for the local M11-L are much lower for plane waves, with a deviation of $0.09 \mathrm{kcal} \mathrm{mol}^{-1}$ between converged values. The error in a Slater basis is about $0.32 \mathrm{kcal} \mathrm{mol}^{-1}$ for the augmented basis, but it is only $0.16 \mathrm{kcal} \mathrm{mol}^{-1}$ for TZ2P. M11-L is the only functional for which the influence of augmentation functions in a Slater basis exceeds the $0.10 \mathrm{kcal}$ $\mathrm{mol}^{-1}$ regime. The largest deviation between aug-cc-pVTZ, 6- 
$311++\mathrm{G}(2 \mathrm{df}, 2 \mathrm{p})$ and the reference value are also observed for M11-L, ranging from 0.45 to $0.69 \mathrm{kcal} \mathrm{mol}^{-1}$.

Overall, for all of the functionals but M06-HF, M11, and M11-L, the results between plane waves, Slater bases, and different Gaussian bases show excellent agreement. The largest deviation is observed in the nonaugmented Slater basis for M06-HF, even though it is still smaller than chemical accuracy $\left(1.0 \mathrm{kcal} \mathrm{mol}^{-1}\right)$. The maximum deviation observed in a Gaussian basis occurs for M11-L/6-311++G(2df,2p), exceeding $0.60 \mathrm{kcal} \mathrm{mol}^{-1}$. Errors of about $0.50 \mathrm{kcal} \mathrm{mol}^{-1}$ can be observed for all bases but not necessarily for the same functional. Maximum deviations in plane waves are reached for M11, whereas the maximum deviation for aug-cc-pVTZ occurs for M11-L. These results highlight the importance of benchmarking the results not only when changing the type of basis, plane waves, Slater, Gaussians, but even when changing from one kind of Gaussian basis (aug-cc-pVTZ) to another $(6-311++G(2 d f, 2 p))$. This can be of particular importance when comparing to results from large benchmarking databases, where sometimes very specific basis sets are used. The deviations observed so far suggest that, for the Minnesota family of functionals, basis-set effects may exceed half a kcal mol${ }^{-1}$. While it is encouraging that these errors are still smaller than chemical accuracy, they can possibly affect the average performance of a functional and conclusions based on benchmarks that cannot be carried out sufficiently close to the basis set limit should bear this source of error in mind. In view of common practice, a careful assessment of basis-set related errors will be of particular importance if a study makes use of a basis set different than the benchmark basis set.

Our plane-wave implementation offers the possibility of smoothly reaching a converged value, as exemplified in the usually small changes of binding energies when increasing $\xi$ from 8 to 12 . The residual differences between the converged values obtained from the custom Gaussian basis by Mardirossian et al. and our converged plane wave results suggest that the flexibility due to the fully delocalized plane waves leaves room for an improved description of the basis-set limit.

4.2. Implications for Reaction Enthalpies of the HC7/ 11 Database. Given the popularity and success of the Minnesota functionals in the description of organic molecules, ${ }^{13,23}$ we now consider their performance for a particularly difficult set of thermochemical data: the reaction enthalpies of the HC7/11 database. ${ }^{22,24}$ In particular for reactions 1 and 2, the introduction of the M06 family constituted a significant improvement over its predecessor, M05.

In order to assess the influence of the choice of basis on the overall performance of the functionals, we have calculated reaction enthalpies at $0 \mathrm{~K}$ for the $\mathrm{HC} 7 / 11$ database of hydrocarbon reactions in a plane wave basis, aug-cc-pVTZ, and the ATZ2P Slater basis for M05 as well as the complete M06 and M11 families. While the standard plane wave setup was a $30 \AA^{3}$ box with $E_{\text {cut }}=125 \mathrm{Ry}$ and $\xi=8$, values for M06, M06$2 \mathrm{X}$, and M06-HF were also calculated in a $20 \AA^{3}$ box with $E_{\text {cut }}$ $=275 \mathrm{Ry}$ and $\xi=12$. These values, however, differ by less than $0.5 \mathrm{kcal} \mathrm{mol}^{-1}$ from those at $E_{\text {cut }}=125$ and are therefore not reported.

4.2.1. The M05 Family. The values for the related M05 and M06 families are given in Table 2. For M05, all values show excellent agreement between plane waves and the atom centered bases. The deviations with respect to the Slater basis range from a minimum of $0.05 \mathrm{kcal} \mathrm{mol}^{-1}$ for reaction 6
Table 2. Reaction Enthalpies for the Members of the HC7/ 11 Database Computed Using the M05, M06, M06-2x, M06$\mathrm{HF}$, and M06-L xc Functional in Plane Waves (125 Ry, $\xi=$ 8), an Augmented Slater basis (ATZ2P), and the Commonly Used Gaussian aug-cc-pVTZ Basis ${ }^{a}$

\begin{tabular}{|c|c|c|c|c|c|}
\hline & system & $\xi=8$ & ATZ2P & $\begin{array}{l}\text { aug-cc- } \\
\text { pVTZ }\end{array}$ & reference \\
\hline \multicolumn{6}{|c|}{ M05 } \\
\hline 1 & E22-E1 & 28.59 & 28.36 & 29.89 & 30.17 \\
\hline \multirow[t]{2}{*}{2} & E31-E1 & 35.60 & 35.46 & 37.30 & 37.68 \\
\hline & E31-E22 & 7.01 & 7.10 & 7.41 & 7.51 \\
\hline 3 & octane isomerization & 7.73 & 7.64 & 7.05 & 7.14 \\
\hline 4 & reaction a & 5.12 & 5.84 & 5.58 & 5.49 \\
\hline 5 & reaction $b$ & 0.16 & 0.45 & 1.22 & 1.00 \\
\hline 6 & reaction $\mathrm{c}$ & 191.17 & 191.12 & 188.55 & 190.75 \\
\hline 7 & reaction $\mathrm{d}$ & 125.11 & 124.81 & 123.61 & 124.99 \\
\hline \multicolumn{6}{|c|}{ M06 } \\
\hline 1 & E22-E1 & 20.96 & 18.46 & 19.65 & 19.26 \\
\hline \multirow[t]{2}{*}{2} & E31-E1 & 27.05 & 24.98 & 26.61 & 26.11 \\
\hline & E31-E22 & 6.09 & 6.52 & 6.96 & 6.85 \\
\hline 3 & octane isomerization & 2.24 & 2.20 & 2.89 & 3.00 \\
\hline 4 & reaction a & 7.32 & 7.48 & 7.77 & 7.89 \\
\hline 5 & reaction $b$ & 13.20 & 13.37 & 14.52 & 14.78 \\
\hline 6 & reaction $\mathrm{c}$ & 190.81 & 192.12 & 196.69 & 198.49 \\
\hline 7 & reaction $\mathrm{d}$ & 124.46 & 125.56 & 129.04 & 130.08 \\
\hline \multicolumn{6}{|c|}{ M06-L } \\
\hline 1 & E22-E1 & 15.71 & 16.45 & 17.59 & 16.28 \\
\hline \multirow[t]{2}{*}{2} & E31-E1 & 18.04 & 19.71 & 21.50 & 19.91 \\
\hline & $\mathrm{E} 31-\mathrm{E} 22$ & 2.33 & 3.26 & 3.91 & 3.63 \\
\hline 3 & octane isomerization & 0.25 & 0.61 & 0.99 & 1.23 \\
\hline 4 & reaction a & 5.70 & 6.07 & 6.08 & 6.23 \\
\hline 5 & reaction $b$ & 8.78 & 9.68 & 10.07 & 10.51 \\
\hline 6 & reaction $\mathrm{c}$ & 179.41 & 185.29 & 191.53 & 190.41 \\
\hline 7 & reaction $\mathrm{d}$ & 115.98 & 120.44 & 124.85 & 124.01 \\
\hline \multicolumn{6}{|c|}{ M06-2X } \\
\hline 1 & E22-E1 & 20.18 & 15.77 & 16.51 & 16.30 \\
\hline \multirow[t]{2}{*}{2} & E31-E1 & 28.39 & 23.78 & 23.94 & 23.65 \\
\hline & E31-E22 & 8.21 & 8.01 & 7.43 & 7.35 \\
\hline 3 & octane isomerization & 1.34 & 1.44 & 1.76 & 2.11 \\
\hline 4 & reaction a & 7.58 & 7.55 & 7.71 & 7.98 \\
\hline 5 & reaction $b$ & 12.65 & 12.70 & 13.28 & 14.02 \\
\hline 6 & reaction $\mathrm{c}$ & 203.77 & 198.24 & 198.46 & 198.36 \\
\hline 7 & reaction $\mathrm{d}$ & 133.81 & 129.60 & 129.64 & 129.44 \\
\hline \multicolumn{6}{|c|}{ M06-HF } \\
\hline 1 & E22-E1 & 21.38 & 13.30 & 13.98 & 14.36 \\
\hline \multirow[t]{2}{*}{2} & E31-E1 & 32.52 & 22.78 & 23.22 & 23.90 \\
\hline & E31-E22 & 11.14 & 9.48 & 9.24 & 9.54 \\
\hline 3 & octane isomerization & 1.34 & 2.22 & 3.85 & 3.62 \\
\hline 4 & reaction a & 7.32 & 7.97 & 8.66 & 8.86 \\
\hline 5 & reaction $b$ & 12.30 & 14.10 & 16.79 & 16.92 \\
\hline 6 & reaction $\mathrm{c}$ & 214.00 & 201.76 & 202.74 & 201.17 \\
\hline 7 & reaction $\mathrm{d}$ & 139.92 & 131.26 & 131.99 & 130.61 \\
\hline
\end{tabular}

${ }^{a}$ Reference values correspond to $6-311+\mathrm{G}(2 \mathrm{df}, 2 \mathrm{p})$, which is the recommended basis for benchmarking in Ref 12 .

to $0.68 \mathrm{kcal} \mathrm{mol}^{-1}$ for reaction 4 and from $0.30 \mathrm{kcal} \mathrm{mol}^{-1}$ for reaction 2 to $2.62 \mathrm{kcal} \mathrm{mol}^{-1}$ for reaction 6 (which is still in the subpercent regime) for aug-cc-pVTZ. However, reactions 5 and 6 show the largest spread over all bases, with results differing by about $0.22 \mathrm{kcal} \mathrm{mol}^{-1}$ between $6-311++\mathrm{G}(2 \mathrm{df}, 2 \mathrm{p})$ and aug-cc-pVTZ for reaction 5 and reaction 6 even showing the best agreement between Slater bases and plane waves 
among all the test set. The difference between plane waves and the Slater basis is only about $0.29 \mathrm{kcal} \mathrm{mol}^{-1}$ for reaction 5 and therefore much lower than the deviation between Slater basis and aug-cc-pVTZ $\left(0.77 \mathrm{kcal} \mathrm{mol}^{-1}\right)$. Overall, best agreement is reached between the Slater basis and plane waves, with deviation between Slater bases or plane waves and Gaussian bases being slightly larger. However, deviations between augcc-pVTZ and 6-311++G(2df,2p) can be equally sizable for systems 6 and 7. Finally it should be noted that for reactions 1 and 2 , the spread is about equal between all the bases considered here. Overall, the reaction enthalpies obtained from the different bases agree very well, making the results reproducible across different approaches.

4.2.2. The M06 Family. The average agreement is slightly inferior for the M06 family. With respect to M05, the spread of the values increases substantially between all the atomcentered bases. While the deviations remain in a subpercent or sub kcal mol ${ }^{-1}$ range for reactions 3 and 4, they can be larger for the remaining reactions. Slater basis results for reactions 1, 2, and 7 differ between 1 and $4 \mathrm{kcal} \mathrm{mol}^{-1}$ from Gaussian results, which themselves exhibit a significant spread of up to $\approx 2 \mathrm{kcal} \mathrm{mol}^{-1}$ for reaction 7 .

For reactions 3-5, M06 shows outstanding agreement between plane wave basis and Slater results, with a minimum deviation of $0.04 \mathrm{kcal} \mathrm{mol}^{-1}$ and a maximum deviation of 0.17 $\mathrm{kcal} \mathrm{mol}^{-1}$. Deviations between plane waves and the Gaussian bases correspond to those observed between ATZ2P and Gaussian functions. The agreement for reactions 1,2, 6, and 7 is less homogeneous, ranging from 1.5 to $2.07 \mathrm{kcal} \mathrm{mol}^{-1}$. The relative errors for reactions 6 and 7, however, do not exceed $2 \%$ with respect to a Slater basis. For reactions 1 and 2, plane wave and Slater basis values show a comparable absolute deviation with respect to the Gaussian bases but are of opposite sign.

Plane wave results for the local M06-L are again closest to those obtained in a Slater basis but consistently lower. In spite of a good overall agreement, deviations between ATZ2P and plane waves can occasionally exceed $1 \mathrm{kcal} \mathrm{mol}^{-1}$. For the isomerization reactions $\mathbf{1}$ and 2 , values show excellent agreement between the Slater ATZ2P and the Gaussian reference basis, whereas the differences between both plane waves and aug-cc-pVTZ with respect to ATZ2P are comparable but of opposite sign. In both cases, the spread of values slightly exceeds chemical accuracy with respect to the reference value. Similarly, the octane isomerization 3 exhibits a large spread, with the value from ATZ2P again lying in between plane waves and aug-cc-pVTZ. In this case, notably, relative errors are large, with the plane wave, Slater basis, and aug-cc-pVTZ values being roughly one, two, and three-quarters of the value obtained in the reference basis. However, due to the small magnitude of the reaction enthalpy, the errors stay below chemical accuracy. Best agreement is reached for reaction 4, with a deviation of $0.37 \mathrm{kcal} \mathrm{mol}^{-1}$ between ATZ2P and plane waves and $0.16 \mathrm{kcal} \mathrm{mol}^{-1}$ between ATZ2P and the reference basis. The spread is again larger for reactions 5,6 , and 7 , but the difference between plane waves/ATZ2P and aug-cc-pVTZ/ATZ2P remain either within the sub-kcal $\mathrm{mol}^{-1}$ range (reaction 5) or lie within 5\% of the reference value. In all three cases, the deviations are again of very similar absolute values but of opposite sign.

M06-2X exhibits a behavior largely reminiscent of M06. Enthalpies for reactions 3-5 agree well between plane waves and ATZ2P, while the deviations for reactions $1,2,6$, and 7 roughly double with respect to M06, although they still exhibit similar trends. M06-HF shows the worst agreement among the M06 family, with deviations up to 1 order of magnitude larger than for M06 itself. For reactions 3-5, however, deviations with respect to a Slater basis remain comparable to those between the Slater basis and the Gaussian reference basis. In general, for the hybrids of the M06 family, the maximum deviation with respect to atom-centered bases increases with the percentage of exact exchange.

Overall, for M05, M06, and M06-L, the enthalpies obtained in plane waves show excellent agreement with the values obtained in an augmented Slater basis. Deviations with respect to Gaussian bases may be somewhat larger, with changes between Slater functions and plane waves often being comparable to a change from Slater functions to Gaussians. For M06-2X and M06-HF, the most important deviations are observed for reactions 1 and 2 . Absolute errors are larger for reactions 6 and 7, but due to the large magnitude of the enthalpy, relative errors remain much lower than for $\mathbf{1}$ and $\mathbf{2}$. However, the enthalpy of the conversion of reactant E22 to product E31, which is not listed as an official database entry, reveals good agreement between the basis sets for all the functionals; for this system, only M06-HF shows a value that exceeds chemical accuracy. This observation indicates that the problem might be linked to reactant E1. The source of this considerable deviations for reactions $\mathbf{1}$ and $\mathbf{2}$ will therefore be examined further on in the text.

4.2.3. The M11 Family. The basis-set dependence of the enthalpies obtained from M11 is comparably uniform for all reactions. For the isomerizations $\mathbf{1}$ and $\mathbf{2}$, the values from the reference Gaussian basis $(6-311+G(2 d f, 2 p))$ lie between the values from ATZ2P/aug-cc-pVTZ and plane wave results, respectively, with deviations ranging from -0.9 to $+0.9 \mathrm{kcal}$ $\mathrm{mol}^{-1}$ with respect to the reference. For the octane isomerization 3, plane wave results deviate by about $0.5-1.0$ $\mathrm{kcal} \mathrm{mol}^{-1}$ from results obtained using Gaussians, whereas the difference between Slater and Gaussian bases exceeds chemical accuracy, ranging from 1.1 to $1.5 \mathrm{kcal} \mathrm{mol}^{-1}$. For reactions 4 and 5, plane waves and ATZ2P show excellent agreement, whereas the values from aug-cc-pVTZ and the Gaussian reference basis lie up to $2 \mathrm{kcal} \mathrm{mol}^{-1}$ higher. Interestingly, the situation is different for reactions 6 and 7, where plane waves and aug-cc-pVTZ agree well; the ATZP and 6-311+G(2df,2p) results are up to $4 \mathrm{kcal} \mathrm{mol}^{-1}$ higher in energy (which corresponds to a $5 \%$ range).

Energetics vary more considerably in the case of M11-L, in analogy with the slow basis set convergence documented in ref 1 and the trends observed in the present work for the HF dimer. For the isomerizations, the enthalpies span a range of over $4 \mathrm{kcal} \mathrm{mol}^{-1}$; ATZ2P values are the lowest, plane wave values the largest, aug-cc-pVTZ and $6-311+\mathrm{G}(2 \mathrm{df}, 2 \mathrm{p})$ lie in between and differ themselves by almost $2 \mathrm{kcal} \mathrm{mol}^{-1}$. For reactions 3-5, all atom-centered bases agree well, with plane wave results deviating by more than $1 \mathrm{kcal} \mathrm{mol}^{-1}$ with respect to the closest Gaussian value. Reactions 6 and 7 show again good agreement within about $1 \mathrm{kcal} \mathrm{mol}^{-1}$ between plane waves and Slater functions, whereas the enthalpies obtained for aug-cc-pVTZ and the reference basis are between 2-3 kcal $\mathrm{mol}^{-1}$ higher. These results highlight the possible high sensitivity of range-separated density functionals to the underlying basis set.

Given the large spread of enthalpies obtained in atomcentered bases, plane wave results still agree well with the other 
basis sets. Consistent with ref 1 , we find that M11-L is more sensitive to basis set effects than its hybrid equivalent, M11. This sensitivity is reflected in a considerable change of reaction enthalpies when changing the type of basis function. While similar trends hold for M11, they are exacerbated for M11-L. M11-L therefore appears to be exceptionally sensitive to the underlying functional form of the basis, which may be due to its dual-range functional form: Similar trends were observed for certain systems when comparing excited states obtained from range-separated functionals in plane waves and Gaussians. ${ }^{6}$ Like for the M06 family, plane waves therefore allow for new insights on converged energetics to be obtained. In view of the improved accuracy of M11 over M06, the fact that converged quantities are readily available from plane waves is particularly favorable.

4.2.4. Summary. Overall, results obtained from plane waves and Slater bases show satisfactory agreement with Gaussian bases, even if there appears to be no systematic trend linking plane wave, Slater, and Gaussian basis results among all of the functionals and systems. While the spread between the results may be considerable for a given reaction, the deviations between the different bases are rather evenly spread for many of the functionals considered here (M05, M06, M06-L, M11, M11-L). Large relative deviations are only observed for the isomerization reactions $\mathbf{1}$ and $\mathbf{2}$. Often, where larger deviations can be observed, Slater basis results lie between plane wave and aug-cc-pVTZ values. In many cases, the deviations of plane waves and aug-cc-pVTZ with respect to the Slater basis are of comparable absolute value but are of opposite sign. In the case of M11, which has a different functional form, values from the Gaussian reference basis lie between those obtained in plane waves and those obtained with Slater functions, which may be attributed to effects due to range-separation. The only significant absolute deviations between plane waves and atom-centered bases occur for M06-2X, M06-HX, and M11L, which, with the exception of M06-2X, have both been identified as difficult to converge in Gaussian bases by Mardirossian et al. ${ }^{1}$ M06-L, on the other hand, appears to be more well-behaved in plane waves than in Gaussians, exhibiting the lowest deviations within the M06 family along with M06 itself. In the case of M11-L, deviations are larger than for M11 but comparable to the differences observed within different atom-centered bases. This indicates that the functional simply calls for a very flexible basis. M05, M06, and M06-L show the best overall agreement across all bases considered here.

Our results show that a plane wave description can add valuable information on the convergence and basis set limit of reaction enthalpies. In particular, where energetics differ considerably between Slater functions and Gaussian basis sets, the fully delocalized plane wave basis allows for a more flexible description and a convenient approach to the basis set limit.

4.3. Energy Density and Exact Exchange Analysis for the M06 Family. For the hybrid functionals of the M06 family, agreement between plane wave and atom-centered basis results for the isomerizations $\mathbf{1}$ and $\mathbf{2}$ noticeably deteriorates with increasing Hartree-Fock exchange contribution. From M06 over M06-2X to M06-HF, the maximum errors increase from 2 over 5 to $14 \mathrm{kcal} \mathrm{mol}^{-1}$. In contrast, the conversion of E31 to E22 is much more accurately described by all the functionals, with a maximum error of about $1.5 \mathrm{kcal} \mathrm{mol}^{-1}$ for M06-HF. The problem therefore appears to be related to the description of reactant E1.
We first consider the effect of exact exchange. The pure Hartree-Fock enthalpy for reaction 1 calculated using the same plane wave setup as used in Table 2 is $-10.29 \mathrm{kcal} \mathrm{mol}^{-1}$, whereas the corresponding value obtained using an atomcentered aug-cc-pVTZ basis is $-12.30 \mathrm{kcal} \mathrm{mol}^{-1}$. While this difference is not negligible, it is well within the spread of values that could be observed for several systems and functionals, even among the two Gaussian basis sets. Therefore, the exact exchange contribution cannot alone account for all of the sizable differences between plane wave and Gaussian values that were obtained using M06-2X and M06-HF.

In order to assess the possible influence of pseudising the core electrons, it appears pertinent to analyze the exchange and correlation energy densities $\epsilon_{\mathrm{x}}(\mathbf{r})$ and $\epsilon_{\mathrm{c}}(\mathbf{r})$, i.e., the integrands of the xc functional

$$
\begin{aligned}
& E_{\mathrm{xc}}=\int \mathrm{d} \mathbf{r} \rho^{4 / 3}(\mathbf{r})\left(K_{\mathrm{x}}(\mathbf{r})+K_{\mathrm{c}}(\mathbf{r})\right) \\
& =\int \mathrm{d} \mathbf{r} \epsilon_{\mathrm{x}}(\mathbf{r})+\epsilon_{\mathrm{c}}(\mathbf{r})
\end{aligned}
$$

for reactant and product of reaction 2 and all of the functionals of the M06 family. Table 4 lists values for $\epsilon$ summed over the nuclear core region, i.e., regions of real space that lie at points $\mathbf{r}_{\text {core }}$ within the cutoff radius $r_{\mathrm{c}}$ of the pseudopotentials employed for a nucleus $\alpha$ at nuclear coordinate R:

$$
E_{\mathrm{xc}}^{\left(r_{\mathrm{c}}\right)}=\sum_{\alpha} \int_{0}^{\mathbf{r}_{\mathrm{c}}} \mathrm{d}\left(\mathbf{r}-\mathbf{R}_{\alpha}\right)\left(\epsilon_{\mathrm{x}}(\mathbf{r})+\epsilon_{\mathrm{c}}(\mathbf{r})\right)
$$

Table 4 shows that irrespective of the differences between plane wave and atom-centered results in Table 3, the changes

Table 3. Reaction Enthalpies for the Members of the HC7/ 11 Database Computed Using the M11 and M11-L xc Functional in Plane Waves (125 Ry, $\xi=8$ ), an Augmented

\begin{tabular}{|c|c|c|c|c|c|}
\hline & system & $\xi=8$ & ATZ2P & $\begin{array}{l}\text { aug-cc- } \\
\text { pVTZ }\end{array}$ & reference \\
\hline \multicolumn{6}{|c|}{ M11 } \\
\hline 1 & $\mathrm{E} 22-\mathrm{E} 1$ & 21.67 & 20.14 & 20.19 & 20.83 \\
\hline 2 & E31-E1 & 29.48 & 28.09 & 27.59 & 28.94 \\
\hline 3 & octane isomerization & 3.52 & 3.00 & 4.10 & 4.52 \\
\hline 4 & reaction a & 6.60 & 6.94 & 7.38 & 7.47 \\
\hline 5 & reaction $\mathrm{b}$ & 13.40 & 13.35 & 15.16 & 15.68 \\
\hline 6 & reaction $\mathrm{c}$ & 193.15 & 196.20 & 193.97 & 198.68 \\
\hline 7 & reaction $\mathrm{d}$ & 125.61 & 128.15 & 126.46 & 129.52 \\
\hline \multicolumn{6}{|c|}{ M11-L } \\
\hline 1 & E22-E1 & 19.99 & 14.60 & 17.12 & 15.91 \\
\hline 2 & E31-E1 & 26.52 & 21.12 & 24.26 & 22.80 \\
\hline 3 & octane isomerization & 0.01 & 1.29 & 1.27 & 1.14 \\
\hline 4 & reaction a & 6.51 & 8.26 & 8.17 & 7.88 \\
\hline 5 & reaction $b$ & 9.69 & 13.60 & 13.56 & 12.82 \\
\hline 6 & reaction $\mathrm{c}$ & 195.12 & 194.15 & 197.70 & 198.55 \\
\hline 7 & reaction $\mathrm{d}$ & 126.83 & 126.52 & 129.47 & 129.74 \\
\hline
\end{tabular}
Slater Basis (ATZ2P), aug-cc-pVTZ, and the Reference Basis $(6-311+G(2 d f, 2 p))$

of the contribution to $\epsilon_{\mathrm{xc}}$ due to the core are below $1 \%$. Similarly, the magnitude of the energy density itself is no indicator of the deviation that is to be expected with respect to all-electron calculations. It is however notable that the exchange-correlation contribution from M06-HF takes on an 
Table 4. Values for $E_{\mathrm{x}}^{\left(r_{\mathrm{c}}\right)}, E_{\mathrm{c}}^{\left(r_{\mathrm{c}}\right)}$, and Their Sum, $E_{\mathrm{xc}}^{\left(r_{\mathrm{c}}\right)}$ around the Nuclear Core Region ${ }^{a}$

\begin{tabular}{|c|c|c|c|c|c|c|}
\hline \multirow[b]{2}{*}{ functional } & \multicolumn{3}{|c|}{ reactant $(\mathrm{E} 1)$} & \multicolumn{3}{|c|}{ product (E31) } \\
\hline & $E_{\mathrm{x}}^{\left(r_{c}\right)}[$ a.u. $]$ & $E_{\mathrm{c}}^{\left(r_{c}\right)}[$ a.u. $]$ & $E_{\mathrm{xc}}^{\left(r_{\mathrm{cc}}\right)}[$ a.u. $]$ & $E_{\mathrm{x}}^{\left(r_{\mathrm{c}}\right)}[$ a.u. $]$ & $E_{\mathrm{c}}^{\left(r_{\mathrm{c}}\right)}[$ a.u.] & $E_{\mathrm{xc}}^{\left(r_{c}\right)}[$ a.u. \\
\hline M05 & -0.2420 & -0.0028 & -0.2438 & -0.2406 & -0.0027 & -0.2432 \\
\hline M06 & -0.1431 & -0.0589 & -0.2020 & -0.1443 & -0.0576 & -0.2019 \\
\hline M06-L & -0.2537 & -0.0208 & -0.2735 & -0.2532 & -0.0199 & -0.2732 \\
\hline M06-2X & -0.1354 & -0.0194 & -0.1547 & -0.1353 & -0.0187 & -0.1540 \\
\hline M06-HF & +0.0130 & -0.0596 & -0.0466 & +0.0137 & -0.0587 & -0.0450 \\
\hline
\end{tabular}

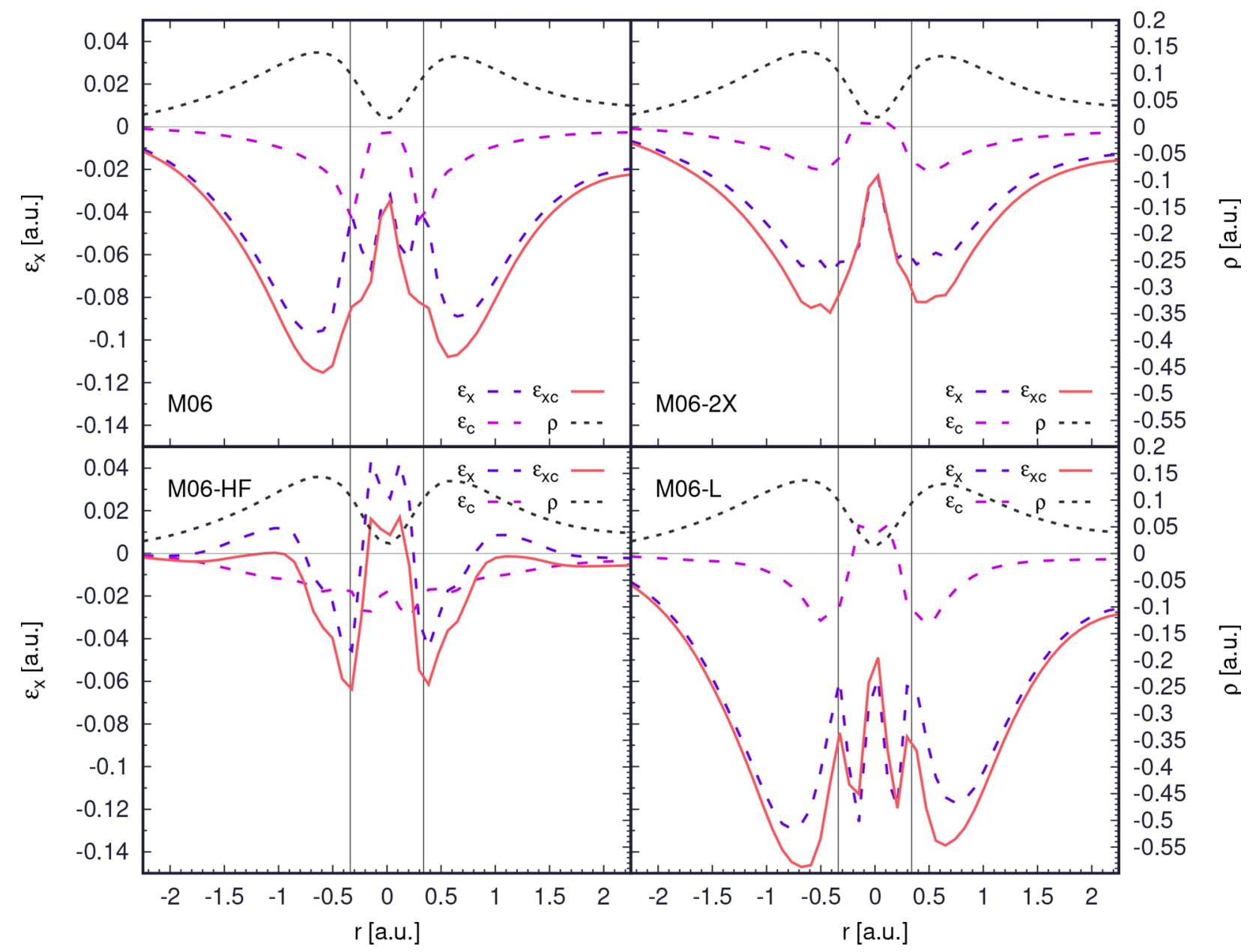

Figure 4. Zoom on the values of the energy density $\epsilon(\mathbf{r})$ for exchange $\mathrm{x}$, correlation c, and exchange-correlation xc for the M06, M06-L, M06-2X, and M06-HF functional at the example of a cut through a carbon-hydrogen bond of product E31 of reaction 2. The electron density is displayed on the secondary $y$ axis.

$\left(\right.$ unphysical $^{1}$ ) positive value which counterbalances the $100 \%$ exact exchange employed in this functional.

In order to assess the effect of pseudisation further, Figure 4 shows the electron density along with the energy density $\epsilon$ for a cut through one of the $\mathrm{C}-\mathrm{H}$ bonds of the reaction product E31. Both exchange and correlation energy densities are smooth outside the core region $r_{c}$ for both M06 and M06-L. Within $r_{\mathcal{c}}$, they can oscillate considerably, which is due to a local maximum in $\tau(\mathbf{r})$ that occurs on top of the carbon nucleus (given that $\tau(\mathbf{r})$ can be viewed as a probe for chemical bonds, one might refer to this as a misdiagnosed chemical bond). Since the oscillations are limited to the pseudised region, no transferability issues are to be expected, which is further illustrated by the values in Table 4. M06-2X and M06-
$\mathrm{HF}$, however, show turning points in $\epsilon_{\mathrm{xc}}$ even outside $r_{\mathrm{c}}$. In particular, $\epsilon_{\mathrm{xc}}$ exhibits a steplike pattern when approaching the cusps of the density, whereas the correlation functional is smoother and more well-behaved. It appears that while the parametrization of M06-2X and M06-HF can be beneficial for weak and long-range interactions, it results in unphysical phenomena when combined with pseudised nuclear cusps. Due to the high flexibility of the plane wave basis, these effects may become particularly prominent. While our results suggests that in many cases these effects do not considerably influence energy differences due to error compensation, this is not necessarily the case for reactions involving reactant E1.

A final quantification of the numerical behavior of the M06 family in a pseudopotential framework can be obtained by 
comparing the integrated $\epsilon_{\mathrm{xc}}$ between reactant and product, where we define

$$
E_{\mathrm{xc}}^{\mathrm{spher}}=\sum_{\alpha} \int_{r_{\mathrm{c}}}^{r_{\mathrm{c}}+\delta r} \mathrm{~d}\left(\mathbf{r}-\mathbf{R}_{\alpha}\right)\left(\epsilon_{\mathrm{x}}(\mathbf{r})+\epsilon_{\mathrm{c}}(\mathbf{r})\right)
$$

as the integral of $\epsilon_{\mathrm{xc}}(\mathbf{r})$ around nuclei $\alpha$, starting from the pseudopotential cutoff radius $r_{\mathcal{c}}$, outside of which the pseudoorbitals are identical to their all-electron counterparts, and integrating up to an upper bound $r_{\max }=r_{\mathrm{c}}+\delta r$. Here, we have chosen the upper bound such that $2 \delta r+r_{\mathrm{c}}^{\alpha}+r_{\mathrm{c}}^{\beta}<d_{\alpha \beta}$ to ensure that there is no double counting between nuclei $\alpha$ and $\beta$ separated by a bond of length $d_{\alpha \beta}$. We then plot the ratio of change of $E_{\mathrm{xc}}^{\text {spher }}$ between reactants and products, $\Delta E_{\mathrm{xc}}^{\mathrm{spher}}$, and the total change in exchange-correlation energy, $\Delta E_{\mathrm{xc}}$ as a function of $r_{\max }$ in Figure 5. The same data is also provided for the exchange-only contribution. For comparison, the values for M05 are given, too.

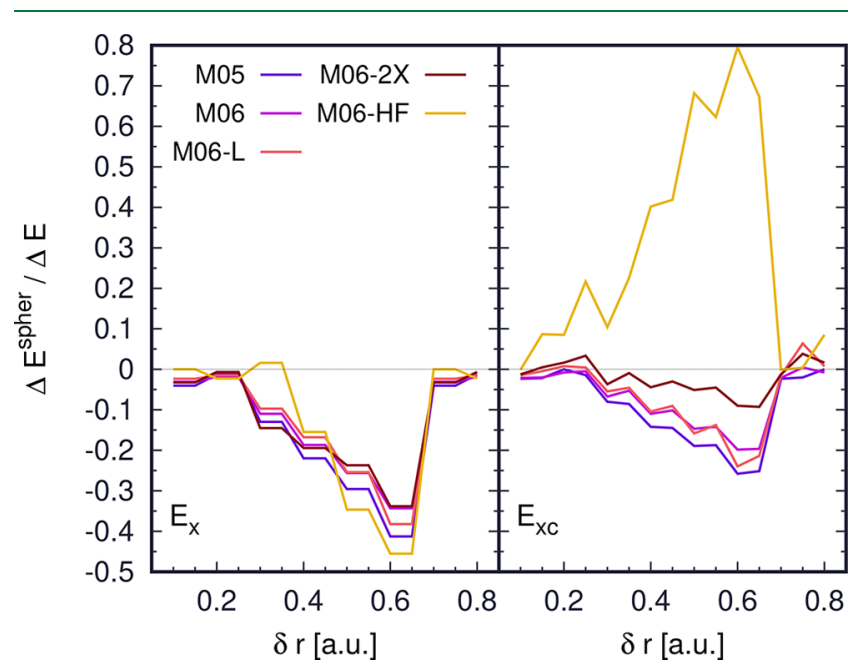

Figure 5. Values for the ratio of change in energy around the nuclei, $\Delta E^{\text {spher }}$, and the total change in energy, $\Delta E$, for exchange only $\mathrm{x}$ as well as exchange-correlation $\mathrm{xc}$ for the M05, M06, M06-L, M06-2X, and M06-HF functional for the isomerization reaction 2.

For the latter, all functionals exhibit negative values for the ratio $\Delta E_{\mathrm{x}}^{\mathrm{spher}} / \Delta E_{\mathrm{x}}$. This implies that the change of $\epsilon_{\mathrm{x}}$ spherically integrated from $r_{\mathrm{c}}$ to $r_{\max }$ around each nucleus is opposite in sign to the overall change of the exchange-correlation energy. This behavior is consistent for all functionals of the M06 family. However, for M06-2X, the ratio increases much slower and reaches lower values, whereas it increases more sharply than for M06-HF. M05, M06, and M06-L show an almost identical behavior. For $\delta r \geq 0.7$, the ratio decreases by almost 1 order of magnitude, indicating that there must be considerable areas where $\Delta e_{\mathrm{x}}(\mathbf{r})>0$, which will compensate for the negative values at $\delta r<0.7$, thus leading to a lower absolute value of the integral. Note that $\delta r \approx 0.7$ is close to the center of a $\mathrm{C}-\mathrm{C}$ or $\mathrm{C}-\mathrm{H}$ bond. For the overall exchange-correlation energy, those trends are even more pronounced. While M06 and M06-L behave almost identically, and M05 very similarly, the ratio is consistently lower in magnitude for M06-2X and reaches positive values for M06-HF. Given that the components of $E_{\mathrm{xc}}$ close to the nuclei counterbalance the overall change in $E_{\mathrm{xc}}$ this can provide a rationale as for why the predicted reaction enthalpy increases in the series M06 $\rightarrow$ M06-2X $\rightarrow$ M06-HF. For M06-2X and M06-HF, the overall contribution close to the nuclei is considerably lower than for the other functionals (which is also illustrated by the values in Table 4 and the $\mathrm{CH}$ bond in Figure 4), the functionals therefore lack some counterbalancing element. A flexible basis such as plane waves may amplify this tendency, resulting in an overestimation of the energy of the isomerization. The different energetics of the Hartree-Fock exchange between all-electron bases and the plane wave/pseudopotential framework can further amplify this behavior.

Figure 6 shows the influence of different basis functions on the electron density at the example of reactant E1. Shown are electron density differences between a converged plane wave density and its analogue obtained using the aug-cc-pVTZ basis for M06 and M06-HF as well as the electron density contour with the same isovalue. In plane waves, for both functionals, the electron density is depleted around the nuclei due to pseudisation, but due to a tight cutoff radius $r_{c}$, those regions are not visible in Figure 6. It can be seen from Figure 6a,c that for M06, residual changes in the electron density occur along $\sigma$ bonds. Their shape is a distinctive result of the higher flexibility in plane waves, leading to small changes of the electron density along bonds. These effects are expected to be highly transferable and to be compensated when comparing different systems. In contrast, Figure 6b,c shows that for M06-HF, plane waves show increased density along all bonds, whereas aug-ccpVTZ yields more density further away from the nuclei. As can be seen from Figure 6a,b, this behavior is amplified around the cyclopropane structure in the case of M06-HF. The large change of enthalpy between plane waves and atom-centered bases correlates with a less localized density difference on E1, in particular around the cyclopropane rings. Other reactions, where agreement between M06-2X, M06-HF, and the atomcentered bases is much better, appear to be far less sensitive to those density differences; in particular, the enthalpies of the "difficult" isodesmic reactions singled out by Grimme ${ }^{24}$ are rather equally predicted by all functionals.
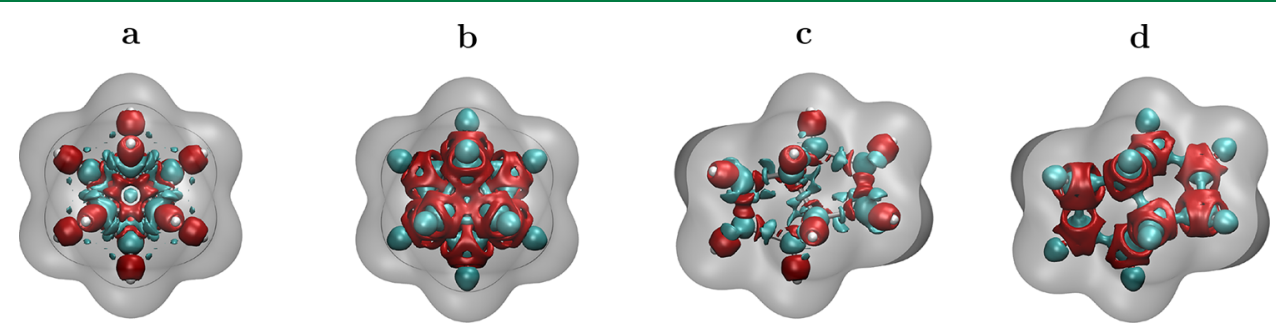

Figure 6. Isosurface $(0.0035 \mathrm{au})$ of the electron density (gray) and the electron density difference between a plane wave setup and aug-cc-pVTZ for compound E1. Regions depicted in red show more density in aug-cc-pVTZ, regions in turquoise have more density in a plane wave basis. Panels a and $\mathrm{c}$ show densities for M06 and panels $\mathrm{b}$ and $\mathrm{d}$ for M06-HF. 
The differences with respect to atom-centered functions are due to the highly flexible nature of plane waves and can be amplified by the varying exact exchange energies predicted in different bases. The features shown in Figures 5 and 6 are a result of the functional form and parametrization of the Minnesota functionals and may only manifest themselves in a highly flexible, delocalized basis such as plane waves.

Plane waves therefore offer a new perspective on the problem of functional convergence. Our results have illustrated that considerable differences with respect to atom-centered systems might occur for some specific functionals and in some particular cases. This is particularly important if results from benchmarks carried out in one basis are to be expected to be transferable to another basis. If the energetics differ considerably between bases, this is reflected in differences in the electron density around the bonding regions and by the ratio of the integral $\Delta E_{\mathrm{xc}}^{\text {spher }} / \Delta E_{\mathrm{xc}}$. Instead, the influence of core-pseudisation has been shown to only negligibly contribute to the energy difference between all-electron and plane wave/ pseudopotential calculations.

\section{COMPUTATIONAL OVERHEAD}

Figure 7 shows the increase in computational time for one DIIS step using a fixed number of 384 CPU cores as a function

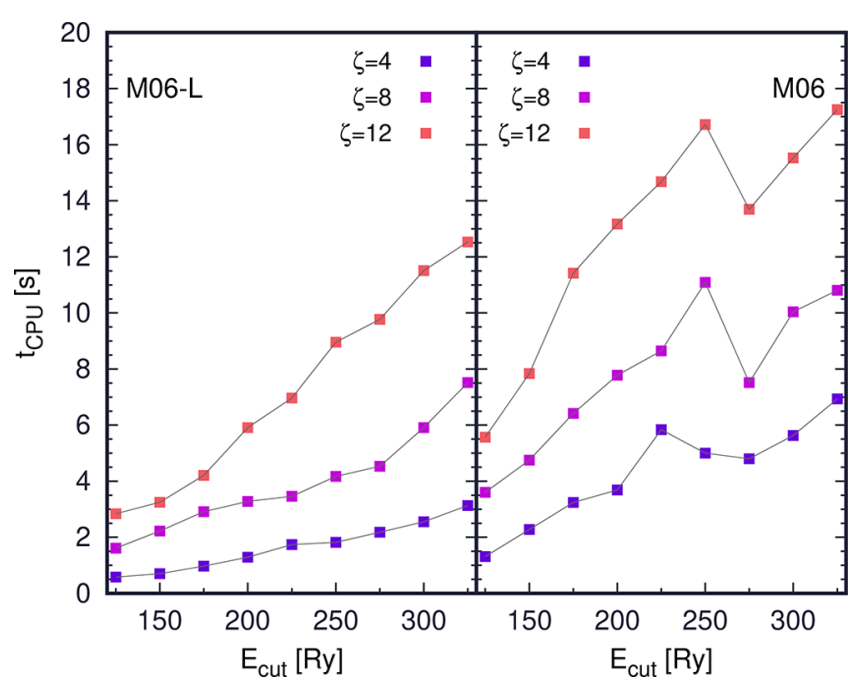

Figure 7. Time per DIIS step for the HF dimer using the M06 and M06-L xc functionals at different cutoff energies and using different values of $\xi$. All calculations were carried out in a $20 \AA \times 15 \AA \times 10 \AA$ box using 384 processors.

of the wavefunction cutoff. While the scaling of the local quantities computed in plane wave codes is in principle linear with an increase in cutoff, here, for certain cutoffs, real space meshes may not be uniformly distributed over processors, resulting in a computational overhead. ${ }^{29}$ In the example of the M06-L functional, it is easily seen that the increase in runtime is proportional to the ratio between $\xi$ used in the calculation and the baseline using $\xi=4$. Since for any meta-GGA, the computational bottleneck is given by the 3D FFTs, an increase in $\xi$ is directly reflected in the run time. The same holds for the hybrid functional M06, although typical load-balancing issues may occur, leading to a nonlinear increase in execution time. This is especially prominent at cutoff energies above $250 \mathrm{Ry}$ and is more pronounced for higher values of $\xi$. Since the Hartree-Fock exchange potential does not have to be calculated on the density grid, an increase in $\xi$ does not affect the run time in a nonlinear manner. Because of the small number of orbitals in the HF dimer, the overhead due to the computation of the exact exchange integrals is modest (about 2.5). The run time of the hybrid-meta GGA in an isolated system and a plane wave basis can further be decreased by resorting to recently proposed density scaling algorithms. ${ }^{58}$

As $\xi=8$ has been found to be sufficient for both the HC7/ 11 database and the HF dimer, the computational overhead of the Minnesota functionals with respect to standard (hybrid) meta-GGA is therefore a factor of 2 . However, the lower orbital cutoff value that can be adopted in combination with a large value of $\xi$ (with respect to the standard choice of $\xi=4$, cf. Figure 3) allows for larger time steps in Car-Parrinello (CP) molecular dynamics, circumventing integration problems for the electronic degrees of freedom associated with highfrequency components in the orbital expansion itself. ${ }^{29}$

\section{CONCLUSIONS}

We have presented a comprehensive analysis of the convergence behavior of the M05-M11 families in a plane wave basis, providing a complete basis set description within a pseudopotential framework. We could demonstrate that given a sufficiently fine integration grid, energy differences for a HF dimer converge rapidly for most functionals with the exception of M06-2X, which requires a particularly high cutoff energy. Results between atom-centered Gaussian and Slater bases agree favorably with those obtained in plane waves. Residual differences were attributed to the high flexibility of the plane wave basis.

In the example of reaction enthalpies of the $\mathrm{HC} / 11$ database, we could subsequently show that for M05, M06, M06-L, M11, and M11-L, there is good agreement between values obtained in plane waves and those obtained from an augmented Slater basis, whereas deviations with respect to augmented Gaussian-type bases may be larger and can exceed chemical accuracy. For the M06-HF and M06-2X functionals, which both include a large percentage of exact exchange, we found that in the case of two isomerizations involving species $\mathrm{E} 1, \mathrm{E} 22$, and E31, changes with respect to atom-centered basis sets can be notable. We have shown that these differences cannot be attributed to core pseudisation. Instead, the difference between exact exchange calculated in a plane wave basis and its atom-centered equivalent can partially account for the differences. Most importantly, sizable deviations between plane waves and atom-centered bases correlate with the ratio between the spherically integrated energy density around the nuclei and the overall exchange-correlation energy, which we have further illustrated in the example of electron density difference maps. For both M06-2X and M06-HF, this ratio has a higher value than in the case of the more well-behaved M06, M06-L, and M05, which all show a more uniform behavior. In particular, for M06, changes in electron density between plane waves and aug-cc-pVTZ were shown to be localized close to the nuclei and $\sigma$ bonds, whereas the electron density differences for M06-HF are substantially more pronounced in regions far from the nuclear core. For the more recent M11 and M11-L, an unusual sensitivity with respect to the kind of basis functions was observed, which was reflected in considerable differences between results obtained with Slater functions and their Gaussian equivalents. We have attributed this to the underlying range-separation, for which the highly flexible plane waves offer a more versatile and complete 
description. These functionals are the most accurate considered in this study and, contrary to Gaussian bases, do not pose convergence problems when used in a plane wave/ pseudopotential framework. Finally, it was shown that the need for a finer integration mesh introduces an overhead of a factor of 2 , independent of the inclusion of exact exchange.

Hence, with this plane wave implementation, it becomes possible to obtain values at the complete basis set limit for the M05, M06, M08, and M11 families. While residual changes with respect to converged Gaussian basis set calculations are small for most systems, they can be sizable for specific reactions described using M06-2X, M06-HF, or M11-L. While these features may remain hidden in a Gaussian basis, they will only surface once functions with an exact asymptotic decay (such as Slater functions) or a fully flexible and delocalized basis (such as plane waves) are used.

\section{ASSOCIATED CONTENT}

\section{S Supporting Information}

The Supporting Information is available free of charge on the ACS Publications website at DOI: 10.1021/acs.jctc.8b00897.

Detailed equations for all members of the M05-M11 families, including the definitions of working variables and notational intricacies (PDF)

\section{AUTHOR INFORMATION}

\section{Corresponding Author}

*E-mail: ursula.roethlisberger@epfl.ch.

\section{ORCID}

Martin P. Bircher: 0000-0002-6905-3130

Ursula Rothlisberger: 0000-0002-1704-8591

\section{Funding}

U.R. gratefully acknowledges financial support from the Swiss National Science Foundation Grant No. 200020-165863 and the NCCR MUST.

\section{Notes}

The authors declare no competing financial interest.

\section{REFERENCES}

(1) Mardirossian, N.; Head-Gordon, M. Characterizing and Understanding the Remarkably Slow Basis Set Convergence of Several Minnesota Density Functionals for Intermolecular Interaction Energies. J. Chem. Theory Comput. 2013, 9, 4453-4461.

(2) Hohenberg, P.; Kohn, W. Inhomogeneous Electron Gas. Phys. Rev. 1964, 136, B864-B871.

(3) Kohn, W.; Sham, L. J. Self-Consistent Equations Including Exchange and Correlation Effects. Phys. Rev. 1965, 140, A1133A1138.

(4) Brunk, E.; Rothlisberger, U. Mixed Quantum Mechanical/ Molecular Mechanical Molecular Dynamics Simulations of Biological Systems in Ground and Electronically Excited States. Chem. Rev. 2015, 115, 6217-6263.

(5) Martell, J. M.; Goddard, J. D.; Eriksson, L. A. Assessment of Basis Set and Functional Dependencies in Density Functional Theory: Studies of Atomization and Reaction Energies. J. Phys. Chem. A 1997, 101, 1927-1934.

(6) Bircher, M. P.; Rothlisberger, U. Plane-Wave Implementation and Performance of á-la-Carte Coulomb-Attenuated ExchangeCorrelation Functionals for Predicting Optical Excitation Energies in Some Notorious Cases. J. Chem. Theory Comput. 2018, 14, 31843195.

(7) Mardirossian, N.; Head-Gordon, M. Thirty years of density functional theory in computational chemistry: an overview and extensive assessment of 200 density functionals. Mol. Phys. 2017, 115, 2315-2372.

(8) Medvedev, M. G.; Bushmarinov, I. S.; Sun, J.; Perdew, J. P.; Lyssenko, K. A. Density functional theory is straying from the path toward the exact functional. Science 2017, 355, 49-52.

(9) Perdew, J. P.; Burke, K.; Ernzerhof, M. Generalized Gradient Approximation Made Simple. Phys. Rev. Lett. 1996, 77, 3865-3868.

(10) Becke, A. D. Density-functional exchange-energy approximation with correct asymptotic behavior. Phys. Rev. A: At., Mol., Opt. Phys. 1988, 38, 3098-3100.

(11) Handy, N. C.; Cohen, A. J. Left-right correlation energy. Mol. Phys. 2001, 99, 403-412.

(12) Zhao, Y.; Truhlar, D. G. The M06 suite of density functionals for main group thermochemistry, thermochemical kinetics, noncovalent interactions, excited states, and transition elements: two new functionals and systematic testing of four M06-class functionals and 12 other functionals. Theor. Chem. Acc. 2008, 120, 215-241.

(13) Peverati, R.; Truhlar, D. G. Quest for a universal density functional: the accuracy of density functionals across a broad spectrum of databases in chemistry and physics. Philos. Trans. $R$ Soc., A 2014, 372, 20120476.

(14) Zhao, Y.; Schultz, N. E.; Truhlar, D. G. Exchange-correlation functional with broad accuracy for metallic and nonmetallic compounds, kinetics, and noncovalent interactions. J. Chem. Phys. 2005, 123, 161103 .

(15) Zhao, Y.; Schultz, N. E.; Truhlar, D. G. Design of Density Functionals by Combining the Method of Constraint Satisfaction with Parametrization for Thermochemistry, Thermochemical Kinetics, and Noncovalent Interactions. J. Chem. Theory Comput. 2006, 2, 364-382.

(16) Zhao, Y.; Truhlar, D. G. A new local density functional for main-group thermochemistry, transition metal bonding, thermochemical kinetics, and noncovalent interactions. J. Chem. Phys. 2006, 125, 194101.

(17) Zhao, Y.; Truhlar, D. G. Density Functional for Spectroscopy: No Long-Range Self-Interaction Error, Good Performance for Rydberg and Charge-Transfer States, and Better Performance on Average than B3LYP for Ground States. J. Phys. Chem. A 2006, 110, 13126-13130.

(18) Peverati, R.; Truhlar, D. G. Improving the Accuracy of Hybrid Meta-GGA Density Functionals by Range Separation. J. Phys. Chem. Lett. 2011, 2, 2810-2817.

(19) Peverati, R.; Truhlar, D. G. M11-L: A Local Density Functional That Provides Improved Accuracy for Electronic Structure Calculations in Chemistry and Physics. J. Phys. Chem. Lett. 2012, 3, 117124.

(20) Zhao, Y.; Truhlar, D. G. Density Functionals with Broad Applicability in Chemistry. Acc. Chem. Res. 2008, 41, 157-167.

(21) Zhao, Y.; Truhlar, D. G. Exploring the Limit of Accuracy of the Global Hybrid Meta Density Functional for Main-Group Thermochemistry, Kinetics, and Noncovalent Interactions. J. Chem. Theory Comput. 2008, 4, 1849-1868.

(22) Zhao, Y.; Truhlar, D. G. Benchmark Databases for Nonbonded Interactions and Their Use To Test Density Functional Theory. J. Chem. Theory Comput. 2005, 1, 415-432.

(23) Zhao, Y.; Truhlar, D. G. A Density Functional That Accounts for Medium-Range Correlation Energies in Organic Chemistry. Org. Lett. 2006, 8, 5753-5755.

(24) Grimme, S. Semiempirical hybrid density functional with perturbative second-order correlation. J. Chem. Phys. 2006, 124, 034108.

(25) Becke, A. D. A new mixing of Hartree-Fock and local densityfunctional theories. J. Chem. Phys. 1993, 98, 1372-1377.

(26) Stephens, P. J.; Devlin, F. J.; Chabalowski, C. F.; Frisch, M. J. $\mathrm{Ab}$ Initio Calculation of Vibrational Absorption and Circular Dichroism Spectra Using Density Functional Force Fields. J. Phys. Chem. 1994, 98, 11623-11627.

(27) Keal, T. W.; Tozer, D. J. Semiempirical hybrid functional with improved performance in an extensive chemical assessment. J. Chem. Phys. 2005, 123, 121103. 
(28) Pulay, P. Ab initio calculation of force constants and equilibrium geometries in polyatomic molecules. Mol. Phys. 1969, 17, 197-204.

(29) Marx, D.; Hutter, J. Ab Initio Molecular Dynamics: Basic Theory and Advanced Methods; Cambridge University Press, 2009.

(30) Valiev, M.; Bylaska, E.; Govind, N.; Kowalski, K.; Straatsma, T.; Van Dam, H. J. J.; Wang, D.; Nieplocha, J.; Apra, E.; Windus, T.; de Jong, W. NWChem: A comprehensive and scalable open-source solution for large scale molecular simulations. Comput. Phys. Commun. 2010, 181, 1477-1489.

(31) Gonze, X.; Jollet, F.; Araujo, F. A.; Adams, D.; Amadon, B.; Applencourt, T.; Audouze, C.; Beuken, J.-M.; Bieder, J.; Bokhanchuk, A.; Bousquet, E.; Bruneval, F.; Caliste, D.; Côté, M.; Dahm, F.; Pieve, F. D.; Delaveau, M.; Gennaro, M. D.; Dorado, B.; Espejo, C.; Geneste, G.; Genovese, L.; Gerossier, A.; Giantomassi, M.; Gillet, Y.; Hamann, D.; He, L.; Jomard, G.; Janssen, J. L.; Roux, S. L.; Levitt, A.; Lherbier, A.; Liu, F.; Lukačević, I.; Martin, A.; Martins, C.; Oliveira, M.; Ponć́, S.; Pouillon, Y.; Rangel, T.; Rignanese, G.-M.; Romero, A.; Rousseau, B.; Rubel, O.; Shukri, A.; Stankovski, M.; Torrent, M.; Setten, M. V.; Troeye, B. V.; Verstraete, M.; Waroquiers, D.; Wiktor, J.; Xu, B.; Zhou, A.; Zwanziger, J. Recent developments in the ABINIT software package. Comput. Phys. Commun. 2016, 205, 106131.

(32) Dunning, T. H. Gaussian basis sets for use in correlated molecular calculations. I. The atoms boron through neon and hydrogen. J. Chem. Phys. 1989, 90, 1007-1023.

(33) Ditchfield, R.; Hehre, W. J.; Pople, J. A. Self-Consistent Molecular-Orbital Methods. IX. An Extended Gaussian-Type Basis for Molecular-Orbital Studies of Organic Molecules. J. Chem. Phys. 1971, 54, 724-728.

(34) Van Lenthe, E.; Baerends, E. J. Optimized Slater-type basis sets for the elements 1-118. J. Comput. Chem. 2003, 24, 1142-1156.

(35) Van Voorhis, T.; Scuseria, G. E. A novel form for the exchangecorrelation energy functional. J. Chem. Phys. 1998, 109, 400-410.

(36) Stoll, H.; Pavlidou, C. M. E.; Preuß, H. On the calculation of correlation energies in the spin-density functional formalism. Theor. Chim. Acta 1978, 49, 143-149.

(37) Hammer, B.; Hansen, L. B.; Nørskov, J. K. Improved adsorption energetics within density-functional theory using revised Perdew-Burke-Ernzerhof functionals. Phys. Rev. B: Condens. Matter Mater. Phys. 1999, 59, 7413-7421.

(38) Perdew, J. P.; Wang, Y. Accurate and simple analytic representation of the electron-gas correlation energy. Phys. Rev. B: Condens. Matter Mater. Phys. 1992, 45, 13244-13249.

(39) Iikura, H.; Tsuneda, T.; Yanai, T.; Hirao, K. A long-range correction scheme for generalized-gradient-approximation exchange functionals. J. Chem. Phys. 2001, 115, 3540-3544.

(40) Tawada, Y.; Tsuneda, T.; Yanagisawa, S.; Yanai, T.; Hirao, K. A long-range-corrected time-dependent density functional theory. $J$. Chem. Phys. 2004, 120, 8425-8433.

(41) Yanai, T.; Tew, D. P.; Handy, N. C. A new hybrid exchangecorrelation functional using the Coulomb-attenuating method (CAMB3LYP). Chem. Phys. Lett. 2004, 393, 51-57.

(42) Chawla, S.; Voth, G. A. Exact exchange in ab initio molecular dynamics: An efficient plane-wave based algorithm. J. Chem. Phys. 1998, 108, 4697-4700.

(43) Gygi, F.; Baldereschi, A. Self-consistent Hartree-Fock and screened-exchange calculations in solids: Application to silicon. Phys. Rev. B: Condens. Matter Mater. Phys. 1986, 34, 4405-4408.

(44) Broqvist, P.; Alkauskas, A.; Pasquarello, A. Hybrid-functional calculations with plane-wave basis sets: Effect of singularity correction on total energies, energy eigenvalues, and defect energy levels. Phys. Rev. B: Condens. Matter Mater. Phys. 2009, 80, 085114.

(45) IBM Corp. www.cpmd.org.

(46) Goedecker, S.; Teter, M.; Hutter, J. Separable dual-space Gaussian pseudopotentials. Phys. Rev. B: Condens. Matter Mater. Phys. 1996, 54, 1703-1710.

(47) Weber, V.; Bekas, C.; Laino, T.; Curioni, A.; Bertsch, A.; Futral, S. Shedding Light on Lithium/Air Batteries Using Millions of Threads on the BG/Q Supercomputer. In 2014 IEEE 28th International Parallel and Distributed Processing Symposium, Phoenix, AZ, May 1923,2014; pp 735-744.

(48) Martyna, G. J.; Tuckerman, M. E. A reciprocal space based method for treating long range interactions in ab initio and force-fieldbased calculations in clusters. J. Chem. Phys. 1999, 110, 2810-2821.

(49) te Velde, G.; Bickelhaupt, F. M.; Baerends, E. J.; Fonseca Guerra, C.; van Gisbergen, S. J. A.; Snijders, J. G.; Ziegler, T. Chemistry with ADF. J. Comput. Chem. 2001, 22, 931-967.

(50) Baerends, E. J.; Ziegler, T.; Atkins, A. J.; Autschbach, J.; Bashford, D.; Baseggio, O.; Bérces, A.; Bickelhaupt, F. M.; Bo, C.; Boerritger, P. M.; Cavallo, L.; Daul, C.; Chong, D. P.; Chulhai, D. V.; Deng, L.; Dickson, R. M.; Dieterich, J. M.; Ellis, D. E.; van Faassen, M.; Ghysels, A.; Giammona, A.; van Gisbergen, S. J. A.; Goez, A.; Götz, A. W.; Gusarov, S.; Harris, F. E.; van den Hoek, P.; Hu, Z.; Jacob, C. R.; Jacobsen, H.; Jensen, L.; Joubert, L.; Kaminski, J. W.; van Kessel, G.; König, C.; Kootstra, F.; Kovalenko, A.; Krykunov, M.; van Lenthe, E.; McCormack, D. A.; Michalak, A.; Mitoraj, M.; Morton, S. M.; Neugebauer, J.; Nicu, V. P.; Noodleman, L.; Osinga, V. P.; Patchkovskii, S.; Pavanello, M.; Peeples, C. A.; Philipsen, P. H. T.; Post, D.; Pye, C. C.; Ramanantoanina, H.; Ramos, P.; Ravenek, W.; Rodríguez, J. I.; Ros, P.; Rüger, R.; Schipper, P. R. T.; Schlüns, D.; van Schoot, H.; Schreckenbach, G.; Seldenthuis, J. S.; Seth, M.; Snijders, J. G.; Solà, M.; M, S.; Swart, M.; Swerhone, D.; te Velde, G.; Tognetti, V.; Vernooijs, P.; Versluis, L.; Visscher, L.; Visser, O.; Wang, F.; Wesolowski, T. A.; van Wezenbeek, E. M.; Wiesenekker, G.; Wolff, S. K.; Woo, T. K.; Yakovlev, A. L. ADF2017; SCM, Theoretical Chemistry, Vrije Universiteit: Amsterdam, The Netherlands, 2017; https://www.scm.com.

(51) Fonseca Guerra, C.; Snijders, J. G.; te Velde, G.; Baerends, E. J. Towards an order-N DFT method. Theo Chem. Acc. 1998, 99, 391403.

(52) Marques, M. A.; Oliveira, M. J.; Burnus, T. Libxc: A library of exchange and correlation functionals for density functional theory. Comput. Phys. Commun. 2012, 183, 2272-2281.

(53) Becke, A. D. A multicenter numerical integration scheme for polyatomic molecules. J. Chem. Phys. 1988, 88, 2547-2553.

(54) Frisch, M. J.; Trucks, G. W.; Schlegel, H. B.; Scuseria, G. E.; Robb, M. A.; Cheeseman, J. R.; Scalmani, G.; Barone, V.; Petersson, G. A.; Nakatsuji, H.; Li, X.; Caricato, M.; Marenich, A. V.; Bloino, J.; Janesko, B. G.; Gomperts, R.; Mennucci, B.; Hratchian, H. P.; Ortiz, J. V.; Izmaylov, A. F.; Sonnenberg, J. L.; Williams-Young, D.; Ding, F.; Lipparini, F.; Egidi, F.; Goings, J.; Peng, B.; Petrone, A.; Henderson, T.; Ranasinghe, D.; Zakrzewski, V. G.; Gao, J.; Rega, N.; Zheng, G.; Liang, W.; Hada, M.; Ehara, M.; Toyota, K.; Fukuda, R.; Hasegawa, J.; Ishida, M.; Nakajima, T.; Honda, Y.; Kitao, O.; Nakai, H.; Vreven, T.; Throssell, K.; Montgomery, J. A., Jr.; Peralta, J. E.; Ogliaro, F.; Bearpark, M. J.; Heyd, J. J.; Brothers, E. N.; Kudin, K. N.; Staroverov, V. N.; Keith, T. A.; Kobayashi, R.; Normand, J.; Raghavachari, K.; Rendell, A. P.; Burant, J. C.; Iyengar, S. S.; Tomasi, J.; Cossi, M.; Millam, J. M.; Klene, M.; Adamo, C.; Cammi, R.; Ochterski, J. W.; Martin, R. L.; Morokuma, K.; Farkas, O.; Foresman, J. B.; Fox, D. J. Gaussian 16, Revision B.01; Gaussian Inc.: Wallingford, CT, 2016.

(55) Tao, J.; Perdew, J. P.; Staroverov, V. N.; Scuseria, G. E. Climbing the Density Functional Ladder: Nonempirical MetaGeneralized Gradient Approximation Designed for Molecules and Solids. Phys. Rev. Lett. 2003, 91, 146401.

(56) Gräfenstein, J.; Izotov, D.; Cremer, D. Avoiding singularity problems associated with meta-GGA (generalized gradient approximation) exchange and correlation functionals containing the kinetic energy density. J. Chem. Phys. 2007, 127, 214103.

(57) Dasgupta, S.; Herbert, J. M. Standard grids for high-precision integration of modern density functionals: SG-2 and SG-3. J. Comput. Chem. 2017, 38, 869-882.

(58) Bircher, M. P.; Rothlisberger, U. Exploiting Coordinate Scaling Relations To Accelerate Exact Exchange Calculations. J. Phys. Chem. Lett. 2018, 9, 3886-3890. 